\title{
Magnetic Nanoparticles
}

\section{Magnetically Active Carbon Nanotubes at Work}

Antoine Stopin, ${ }^{[a]}$ Florent Pineux ${ }^{[a]}$ Riccardo Marega, ${ }^{[a]}$ and Davide Bonifazi ${ }^{*[a, b]}$

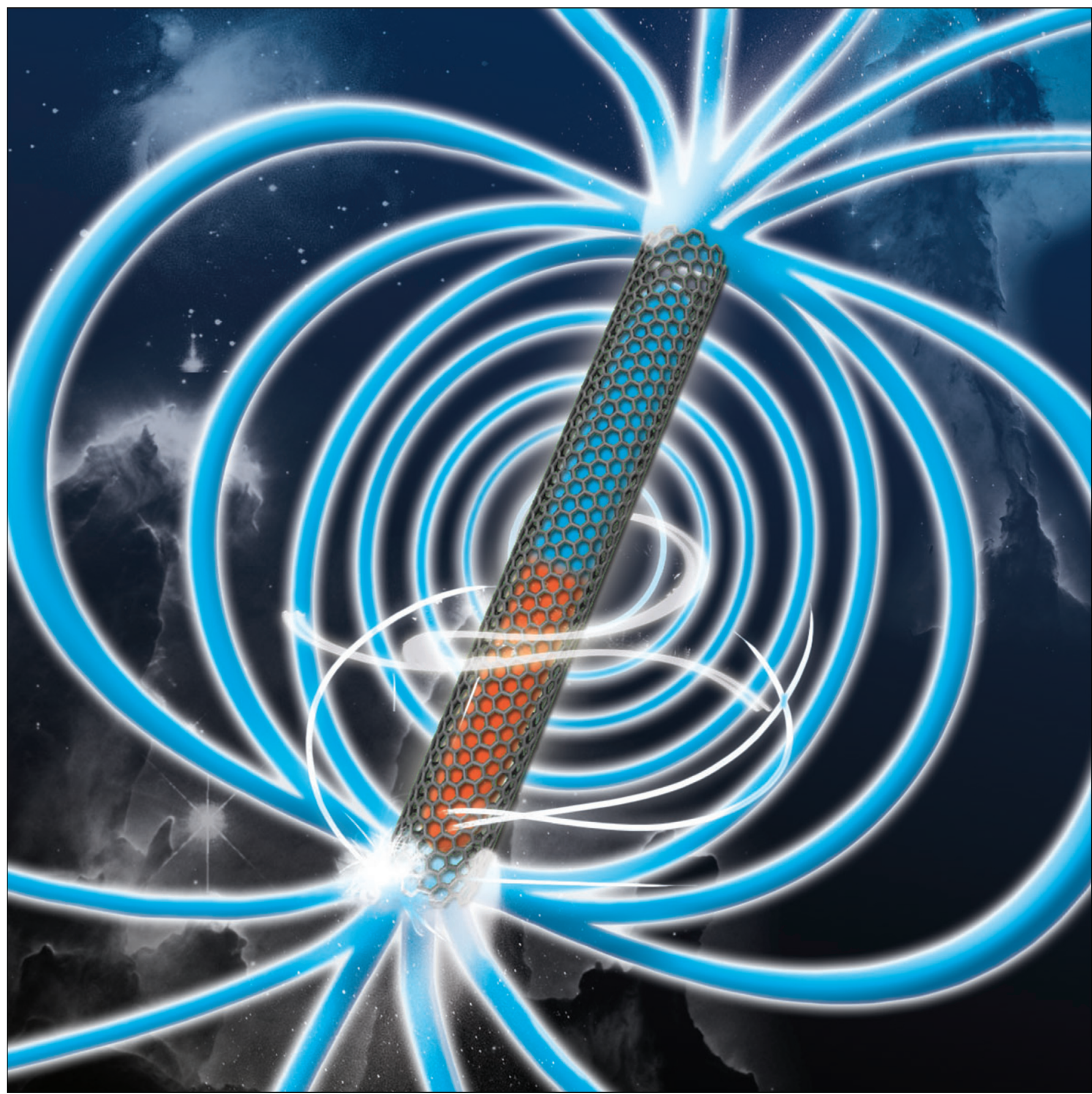


Abstract: Endohedral and exohedral assembly of magnetic nanoparticles (MNPs) and carbon nanotubes (CNTs) recently gave birth to a large body of new hybrid nanomaterials (MNPs-CNTs) featuring properties that are otherwise not in reach with only the graphitic or metallic cores themselves. These materials feature enhanced magnetically guided motions (rotation and translation), magnetic saturation and coercivity, large surface area, and thermal stability. By guiding the reader through the most significant examples in this Concept paper, we describe how researchers in the field engineered and exploited the synergistic combination of these two types of nanoparticles in a large variety of current and potential applications, such as magnetic fluid hyperthermia therapeutics and in magnetic resonance imaging to name a few.

\section{Introduction}

\subsection{General properties and applications of carbon nano- tubes}

In 1991, lijima reported for the first time the targeted synthesis of "helical microtubules of graphitic carbon" constituted of rolled-up layers of graphene, named carbon nanotubes (CNTs), which can be single- or multi-walled (SWCNTs and MWCNTs). ${ }^{[1]}$ The tubes diameter vary from 0.4 to $100 \mathrm{~nm}$ and their length can reach up to the centimeter scale, making CNTs the materials with the highest aspect ratio ever reported. ${ }^{[2]}$ Their unique tubular graphitic structure gives them a large variety of physical properties, such as exceptional stiffness (Young's modulus of SWCNTs is in the order of $1.8 \mathrm{TPa})^{[3]}$ and tensile strength (up to $63 \mathrm{GPa}$ for a single MWCNT), ${ }^{[4]}$ remarkably high axial electrical conductivity (up to $6 \times 10^{6} \mathrm{~A} \mathrm{~cm}^{-2}$ ), ${ }^{[5]}$ helicity-dependent farultraviolet and far-infrared region absorption, ${ }^{[6]}$ and excellent anisotropic thermal conduction and insulating character in the axial $^{[7]}$ and radial directions, ${ }^{[8]}$ respectively. For instance, CNTs have been exploited as reinforcing components in polymeric matrixes to enhance the electrical conductivity of bulk materials at very low mass percentage $(<0.1 \mathrm{wt} \%){ }^{[9]}$ This acquired practical significance after the great efforts devoted to the study of the chemical reactivity of CNTs. ${ }^{[10]}$ Inspired by the organic and inorganic functionalization methodologies previously developed for fullerenes, ${ }^{[11]}$ a broad range of covalent or non-covalent derivatization protocols have been developed for CNTs to 1) improve their processability in organic and aqueous solvents and 2) impart targeted functionalities. ${ }^{[12]}$ This allowed

[a] Dr. A. Stopin, ${ }^{+}$F. Pineux, ${ }^{+}$Dr. R. Marega, Prof. Dr. D. Bonifazi Department of Chemistry and Namur Research College (NARC) University of Namur, Rue de Bruxelles 61, 5000 Namur (Belgium) E-mail:davide.bonifazi@unamur.be

[b] Prof. Dr. D. Bonifazi

Department of Pharmaceutical and Chemical Sciences and INSTM UdR Trieste, University of Trieste Piazzale Europa 1, 34127 Trieste (Italy)

$\left.{ }^{[}{ }^{+}\right]$These authors equally contributed to this work. the scientific community to explore a wide variety of applications spamming from materials ${ }^{[13]}$ to biomedical ${ }^{[14]}$ technologies, such as field-effect transistors (FET) ${ }_{r}^{[15]}$ tunable optoelectronic devices, ${ }^{[16]}$ molecular sensors, ${ }^{[17]}$ and nanocarriers for drugs, $^{[18]}$ proteins, ${ }^{[19]}$ or nucleic acids. ${ }^{[20]}$

After the seminal report from Ajiki et al., ${ }^{[21]}$ who first predicted the magnetic properties of CNTs, the scientific community devoted lot of attention to the fundamental understanding and quantification of the intrinsic magnetic features of SWCNTs and MWCNTs. ${ }^{[22]}$ This fostered a series of subsequent investigations, including the blending of CNTs with other magnetic nanoparticles (e.g., Fe, $\mathrm{Co}, \mathrm{Ni}$ and their oxides, to cite a few) to boost their responses to an external magnetic field. In this respect, the control of the magnetic properties of CNT hybrids is not only important from a fundamental point of view, but also for practical applications, as anisotropy could be for instance exploited for engineering organized functional materials in which CNTs are spatially aligned or driven in a controlled orientation following a remote stimulus. ${ }^{[23]}$ Thus, in this Concept paper we summarize the most recent reports describing the preparation, properties, and exploitation of CNT hybrids bearing magnetic nanoparticles, highlighting the synergistic effects obtained by the combination of these unique nanostructures.

\subsection{Basic concepts and intrinsic magnetic properties of CNTs}

Magnetization describes the way a material modifies and responds to an external magnetic field and can be used to estimate consequential magnetic forces. ${ }^{[2]}$ This value is nothing but the vectorial sum of proton and electron magnetic dipole moments contained in a determined volume of matter. These magnetic dipole moments are associated with spins and orbital motions. ${ }^{[25]}$ Magnetic susceptibility $\chi$ of a material describes the variation of magnetization $M$ as a function of an external magnetic field $H$ [Eq. (1)]. ${ }^{[26,27]}$

$M=\chi H$

In general, materials are categorized as paramagnetic $(\chi>0$, with $M$ adding to $H$ ) or diamagnetic $(\chi<0, M$ opposing to $H$ ) as function of their magnetic susceptibility. ${ }^{[28,29]}$ Magnetic susceptibility values of CNTs depend on their helicity and on the angle between the magnetic field and the tube main axis. Specifically, semiconducting and metallic SWCNTs display higher $\chi$ values when the nanotube develops parallel $\left(\chi^{\|}\right)$to the incident magnetic field rather than perpendicular to it $\left(\chi^{\perp}\right)$. This difference between the parallel and perpendicular responses $\left(\chi^{\|}-\chi^{\perp}\right)$ is an assessment of the anisotropic character of the magnetic susceptibility of CNTs. Fujiwara et al. were the first to study the deposition of individualized MWCNT dispersions onto glass substrates exposed to various magnetic fields, showing a preferential distribution of the tubes along the magnetic field lines. ${ }^{[22 b]}$ This allowed the estimation of the small, yet significant, $\chi$ anisotropy values of $9 \pm 5 \times$ $10^{-6} \mathrm{emu} \mathrm{mol}^{-1}$ (referred to the content of carbon atoms) for MWCNTs. Later, Zaric et al. used magnetophotoluminescence measurements on micelle-dispersed CNTs, suggesting suscepti- 
bility anisotropy values of about $1.5 \times 10^{-5} \mathrm{emu} \mathrm{mol}^{-1}$ for semiconducting SWCNTs with a diameter of $1 \mathrm{~nm} \cdot{ }^{[22 \mathrm{~d}]}$ More recently, Searles et al. used the same method for metallic SWCNTs, deducing $\chi$ values of $3-4 \times 10^{-5}$ emu mol $^{-1}$ in agreement with those theoretically predicted (Figure 1). ${ }^{[22 \mathrm{e}]}$
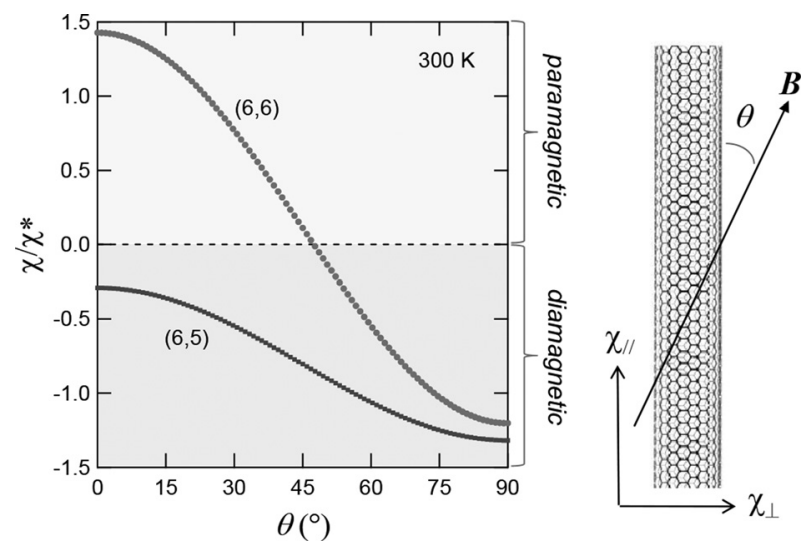

Figure 1. Predicted magnetic susceptibility $\chi$ of $(6,6)$ (metallic) and $(6,5)$ (semi-conducting) SWCNTs as a function of the angle $\theta$ between the CNT main axis and the magnetic field $B$ as calculated by Searles et al. ${ }^{[22 e]}$ Metallic CNTs can be paramagnetic or diamagnetic according to their orientation in the magnetic field whereas semi-conductive CNTs are predicted to be diamagnetic under any conditions. In both cases, their magnetic susceptibility anisotropy $\Delta \chi=\chi_{\|}-\chi_{\perp}$ is higher than 0 , thus sufficient to direct their alignment in a magnetic field. $\chi^{*}=1.46 \cdot 10^{-4}$ emu mol $^{-1}$. Reproduced with permission from reference [22e].

Exploiting the intrinsic $\chi$ anisotropy of MWCNTs, we have reported the first example of an anisotropic fluorescent, thermoresponsive, hydrogel-containing MWCNT. ${ }^{[30]}$ Specifically, we have demonstrated that the control of the alignment of the CNT frameworks induced by a magnetic field (10 T) anisotropically affected the emission properties of CNTs exohedrally decorated with an anionic Eu'll-based complex through ion-pairing interactions established with a water-soluble polycationic polymers (Figure 2). Exploiting the thermoresponsive optical properties of the hydrogel matrix, stimuli-induced switching of the luminescence output could also be reversibly achieved, without affecting the alignment of the CNTs. However, due to the weak anisotropic character of the intrinsic magnetic suscepti- bility of CNTs, their efficient alignment could only be obtained under extremely high magnetic fields (above $10 \mathrm{~T}$ ). Therefore, the small $\chi$ values (from -2 to $+2 \times 10^{-4}$ emumol $^{-1}$ for SWCNTs) ${ }^{[22 e]}$ of pristine CNTs suggest that their exploitation in magnetic resonance imaging or magnetic fluid hyperthermia will remain limited if not intimately combined with other magnetically active nanostructures (see below).

\subsection{Magnetic properties of magnetic nanoparticles: ferro- magnetism and superparamagnetism}

Among all magnetic systems, ferro- and superparamagnetic ${ }^{[31]}$ nanoparticles (MNPs) have found a prominent role as functional materials $s^{[32]}$ in a number of diverse technological applications, such as data recording, ${ }^{[33]}$ catalysis, ${ }^{[34]}$ magnetic resonance imaging ${ }^{[35]}$ magnetic fluid hyperthermia, ${ }^{[36]}$ and magnetically-driven drug delivery. ${ }^{[37]}$ These applications are rendered possible by the combination of their very high responses to external magnetic fields and the possibility to generally tune their magnetic properties as a function of their morphology at the nanoscale. In particular, above a critical size (mainly depending on the aspect ratio and chemical composition), ${ }^{[29,38]}$ a hefty amount of magnetization (called coercive field $H_{c}$ ) is conserved even in the absence of an external magnetic field. Below these critical dimensions, MNPs become superparamagnetic. Namely their magnetization can be randomized by thermal energy, cancelling coercivity while maintaining ferromagnetic susceptibility. These features make MNPs privileged materials to be coupled with CNTs, giving birth to tailored magnetic hybrids with characteristics that both components do not individually possess, thus extending the scope of applications devoted to CNTs and MNPs. ${ }^{[39]}$ For the sake of clarity, we will use the notation MNPs-CNTs when it is generally referred and MNPs@CNTs or MNPs/CNTs when the nanoparticles are either encapsulated or exohedrally grafted.

\section{Preparation of Magnetically Active Carbon Nanotubes}

The main strategies used to prepare MNPs-CNTs hybrids can be divided in two categories: the in-situ and ex-situ methods. a)

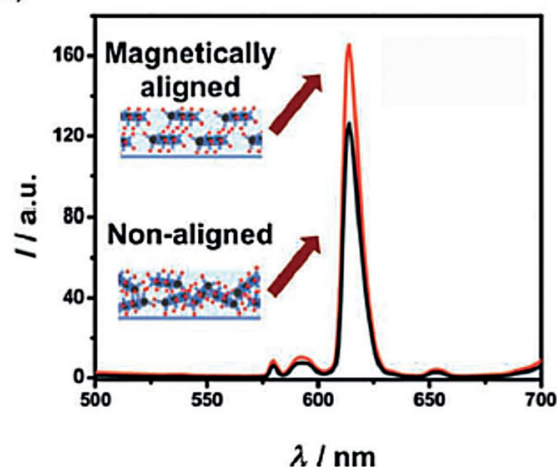

b)

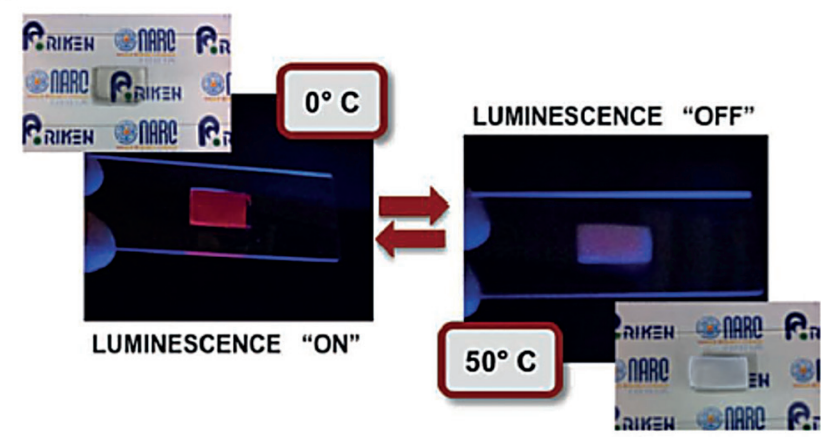

Figure 2. a) Emission spectra $\left(\lambda_{\text {exc }}: 370 \mathrm{~nm}\right)$ of magnetically aligned and non-aligned CNTs.Eu'll hydrogels; b) pictures of the magnetically-aligned thin films of CNTs.Eu"I at 0 and $50^{\circ} \mathrm{C}$ under a $365 \mathrm{~nm}$ light excitation. Reproduced with permission from reference [30]. 

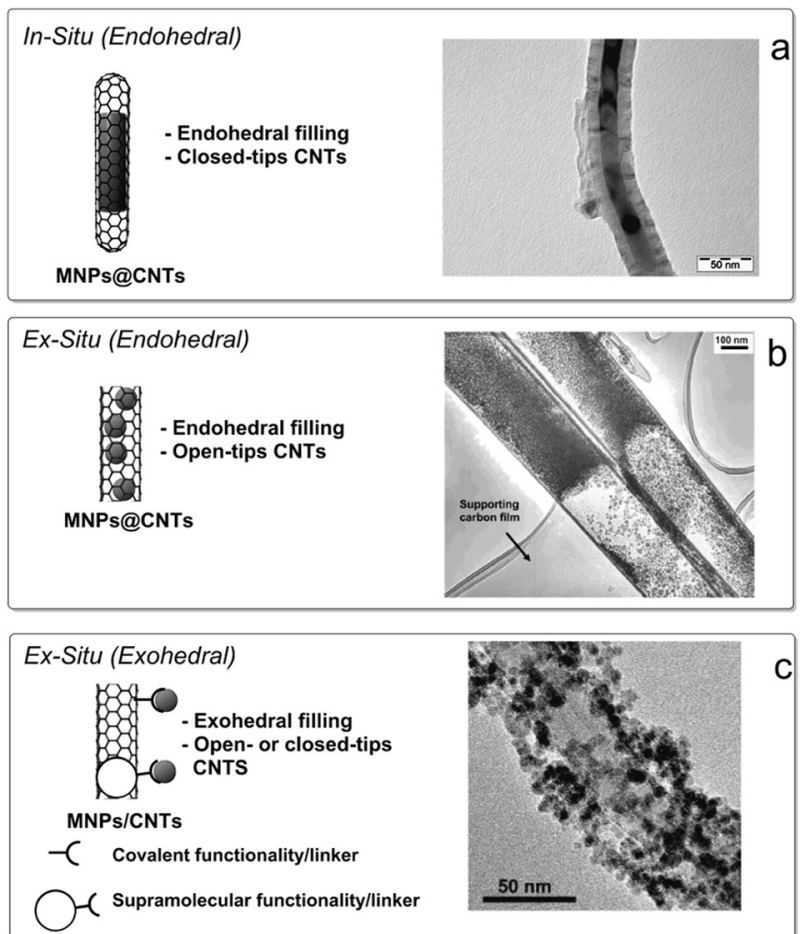

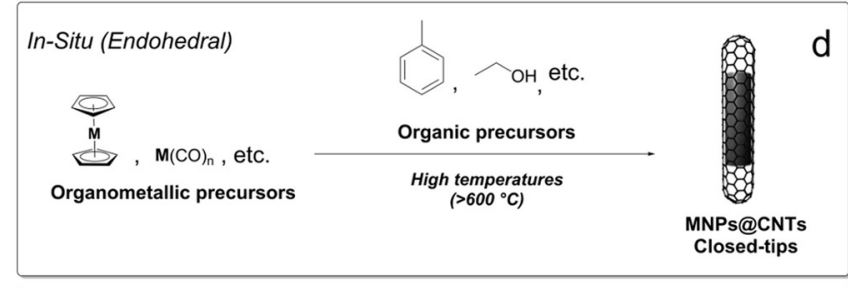

Ex-Situ (Endohedral)
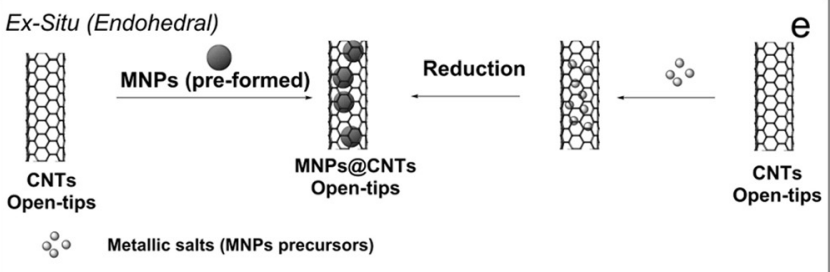

Ex-Situ (Exohedral)
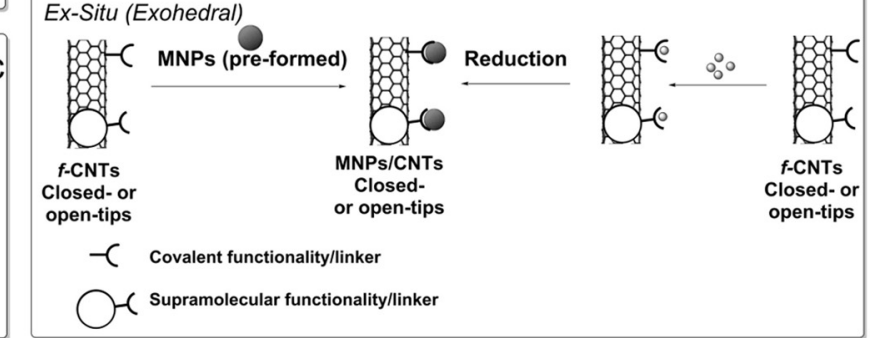

Figure 3. Representative TEM images of different MNP-CNT hybrids: MNPs@CNTs produced by a) in-situ and b) ex-situ protocols, c) MNPs/CNTs produced by ex-situ exhohedral functionalization of CNTs. Scheme of the preparative protocols for MNPs-CNTs by d) in-situ CVD processes and e) ex-situ protocols. TEM images in b) and c) are reproduced with permission from references [40] and [41], respectively.

In the in-situ protocols, formation of MNPs and CNTs is simultaneous, as MNPs act as catalysts for the CNT synthesis, giving endohedrally encapsulated materials (MNPs@CNTs). On the other hand, in the ex-situ protocols, MNPs are added to preformed CNTs, either by filling the endohedral cavity of opentips CNTs (endohedral functionalization, MNPs@CNTs) or by addition onto their external surface (i.e., exohedral functionalization, MNPs/CNTs, Figure 3).

\subsection{In-situ protocols}

Soon after the first arc-discharge production of CNTs by lijma, ${ }^{[1]}$ Bethune et al. serendipitously discovered that metallic Co nanoparticles can act as catalysts for SWCNT synthesis. ${ }^{[42]}$ They noted that Co NPs were enclosed in carbon soot at the base of the SWCNTS and that the powder produced was ferromagnetic. This could be considered as the first, yet unintended, in-situ synthesis of "magnetic CNTs". This led few years later to the development of a gram-scale ${ }^{[43]}$ protocol for the production of SWCNTs by arc-discharge (using $\mathrm{Ni}$ as catalyst) and of highquality SWCNTs by laser-ablation (using this time a mixture of $\mathrm{Ni}$ and Co catalysts). ${ }^{[44]}$ Based on these discoveries, the industrial production of CNTs by the chemical vapor deposition (CVD) method could easily be developed. ${ }^{[45]}$ The CVD process relies on the pyrolysis at high temperatures (generally between 600 and $1300^{\circ} \mathrm{C}$ ) of carbon feedstock (e.g., acetylene, ${ }^{[46]}$ ethylene, ${ }^{[47]}$ benzene, ${ }^{[48]}$ or ethanol ${ }^{[49]}$ ) onto a substrate coated with the catalyst as metallic particles. The generally accepted mechanisms for the formation of CNTs include the hydrocarbon de- composition at the surface of the metal, the carbon diffusion through the metal phase, and the final CNT precipitation either toward the top ("base-growth mechanism") or toward the surface, pushing the metallic particle up ("tip-growth" mechanism) ${ }^{[50]}$ The process stops when the catalyst particle is sequestered inside the nanotube cavity (Figure 4). The currently used transition-metal catalysts for the CVD synthesis are Co, $\mathrm{Fe}$, and $\mathrm{Ni}$, as they can interact with carbon atoms at high temperatures, forming a few carbide phase ${ }^{[51]}$ that possess high carbon diffusion rates. ${ }^{[52]}$ Interestingly, these three metals are also the only (non-alloyed) ferromagnetic materials at room temperature. ${ }^{[53]}$ Since residual amounts of catalyst often

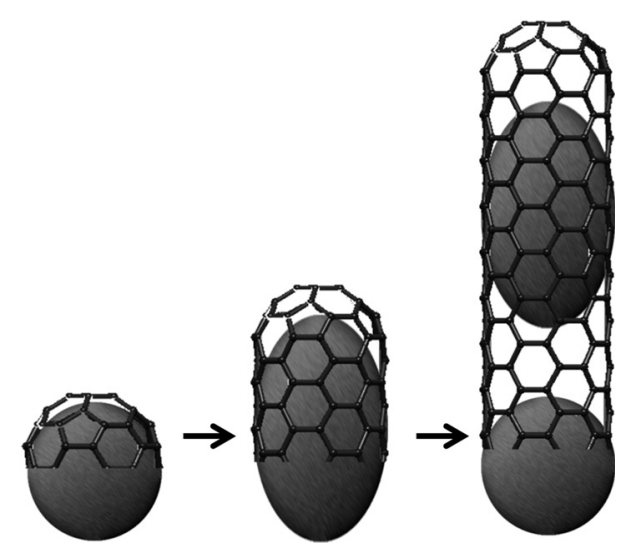

Figure 4. Proposed mechanism for the formation of metal-filled MWCNTs by CVD. 
remain after the synthesis, the vast majority of the as-produced CNTs encapsulate MNPs (MNPs@CNTs). However, these CNTs contain limited amounts of MNPs (generally below $5 \mathrm{wt} \%)$ that are often not sufficient to be efficiently exploited for magnetic applications, like magnetic fluid hyperthermia.

High loading of MNPs can be achieved by complementary addition of organometallic species, like metallocenes (such as ferrocene or cobaltocene) or metal carbonyl complexes (e.g. $\left.\left[\mathrm{Fe}(\mathrm{CO})_{5}\right]\right)$, to the carbon feedstock. The organometallic reagent covers a double role: 1) forms in-situ floating catalytic MNPs during the pyrolysis (i.e., "floating catalyst $\left(\mathrm{CVD}^{\prime \prime}\right.$ ) and 2) acts as molecular precursor for the metal to be encapsulated. ${ }^{[54]}$ Seminal developments have been achieved by Rao and co-workers, ${ }^{[48,55]}$ who showed that different morphologies (diameters, lengths) and metal loading could be obtained by tuning the experimental conditions, such as temperature, deposition time, and hydrocarbon sources. ${ }^{[56]}$

The highest metal loadings of MNPs@CNTs were achieved by the use of ferrocene as a sole source of both carbon and iron atoms yielding Fe@CNT hybrids with metal-filling ratio up to $35 \%$ in weight, featuring the highest values of magnetic saturation $^{[57]}$ (up to $50 \mathrm{emug}^{-1}$ ) for such materials. ${ }^{[58]}$ Along this line, Liu et al. ${ }^{[58 b]}$ also demonstrated that sublimation temperature exerts a dramatic influence on the morphological characteristics of the Fe-containing graphitic materials. In a recent work, Dillon et al. ${ }^{[58 a]}$ showed that Fe@CNTs prepared under different sublimation and pyrolysis temperatures display singular magnetic properties. While the coercivity results connected to the pyrolysis temperature, the magnetic saturation is influenced by the sublimation temperature. ${ }^{[58 b]}$ Metal oxide nanoparticles could also be encapsulated by in-situ protocols. An elegant example has been proposed by Jang and Yoon ${ }^{[59]}$ who described the carbonization of a metal-impregnated polymer precursor for preparing CNTs filled with iron oxide ( $\mathrm{Fe}_{y} \mathrm{O}_{x} @ \mathrm{CNTs}$ ).

\subsection{Ex-situ protocols}

\subsubsection{Endohedral functionalization}

Following the seminal work of Smith et al. demonstrating the possibility of ex-situ endohedral filling of open-tips CNTs with $\mathrm{C}_{60}$ (i.e., CNT peapods), ${ }^{[60]}$ the inner tubular cavity has been used as a host for a wide range of molecular guests. ${ }^{[61]}$ Classical preparative steps for the ex-situ endohedral functionalization usually consist of 1) an oxidative tip-opening, ${ }^{[62]}$ 2) CNT impregnation with a fluid containing the desired molecular species to be encapsulated ${ }^{[63]}$ and, seldom 3 ) thermal closing of the tubes termini. ${ }^{[64]}$ It is important to note that the selective endohedral filling during the impregnation step can be achieved thanks to capillary forces. In particular, it has been proven that the surface tension $(\gamma)$ of the fluid has to be lower than $190 \mathrm{mN} \mathrm{m}^{-1}$ to allow wetting and CNT filling. ${ }^{[65]}$ This includes water $(\gamma=$ $\left.72 \mathrm{mNm}^{-1}\right)$ and most of the organic solvents ( $\gamma$ below $72 \mathrm{mN} \mathrm{m}^{-1}$ ). This approach has also been applied as a complementary method for preparing MNPs@CNTs. In this case, the filling fluid consists of either a dispersion of pre-synthesized MNPs or a solution containing a metallic precursor that under- goes cavity-confined transformation, yielding the encapsulated MNPs. Although requiring more steps than in-situ protocols, ex-situ methods generally allow a better control of the MNP morphology. However, when the CNTs are left open, enclosed MNPs are partially or fully oxidized, thus featuring lower magnetization than that of the metallic analogues.

To the best of our knowledge, the first ex-situ preparation of MNPs@CNTs has been reported by Pham-Huu et al. By filling open-tips CNTs with an aqueous solution of $\mathrm{Fe}\left(\mathrm{NO}_{3}\right)_{3}$ and $\mathrm{Co}\left(\mathrm{NO}_{3}\right)_{2}$, magnetic $\mathrm{CoFe}_{2} \mathrm{O}_{4}$ nanowires were formed at CNT tips under mild thermal treatment, as low as $50^{\circ} \mathrm{C} .{ }^{[67]}$ In a further study, Tessonier et al. demonstrated the possibility to reduce these encapsulated $\mathrm{CoFe}_{2} \mathrm{O}_{4}$ nanowires into CoFe particles with $\mathrm{H}_{2}$ at $400{ }^{\circ} \mathrm{C}$ for $2 \mathrm{~h}^{[68]}$ Interestingly, the rodlike morphology was retained thanks to the mild reduction conditions. More recently, CNTs have been also exploited by Baaziz et al. for encapsulating monodisperse $50 \mathrm{~nm}$-diameter MNPs of Co (Figure 5). ${ }^{[6]]}$ For this purpose, cobalt stearate impregnated in

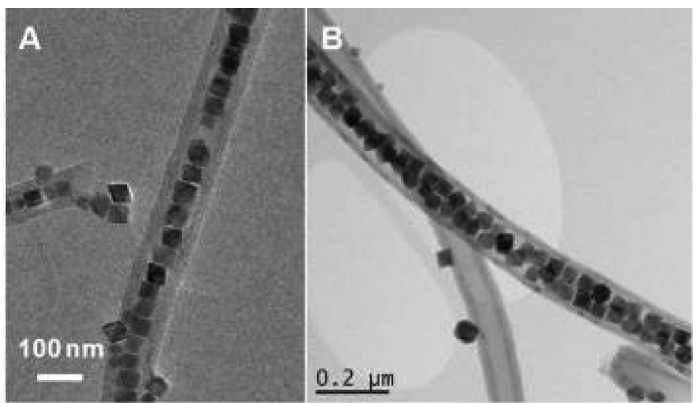

Figure 5. TEM pictures of Co-based MNPs@CNTs prepared by thermal decomposition of cobalt stearate. Reproduced with permission from reference [66].

open-tips CNTs was used as a precursor for the thermal transformation reaction. ${ }^{[32 a, 69]}$ Reference thermal reduction experiments in the absence of the carbon frameworks showed the limited formation of NPs thus confirming the templating role exerted by the nanotube cavity on the nanoparticle synthesis. Another interesting work reports the exploitation of molten salts as both CNT fillers ${ }^{[70]}$ and MNP precursors. As shown by Tîlmaciu et al., ${ }^{[71]}$ molten $\mathrm{Fel}_{2}\left(T_{\mathrm{f}}=587^{\circ} \mathrm{C}\right)$ has been used to fill open-tips double-walled CNTs (DWCNTs) at $690^{\circ} \mathrm{C}$ to yield, after subsequent reaction (with $\mathrm{H}_{2}$ ), and oxidation (in air) steps, CNT-confined $\gamma-\mathrm{Fe}_{2} \mathrm{O}_{3}$ superparamagnetic NP chains.

Following an alternative avenue, Korneva et al. reported the first encapsulation of pre-formed MNPs. ${ }^{[40]}$ In this approach, CNTs were grown inside the pore of an alumina matrix by CVD and a ferrofluid containing $\mathrm{Fe}_{3} \mathrm{O}_{4}$ MNPs was guided inside the CNTs through the action of a permanent magnet. The desired MNPs@CNTs material was obtained by removing the carrier fluid and the alumina support (Figure 6). This strategy allowed for the preparation of CNTs filled with structurally monodisperse MNPs. Along the same vein, Khlobystov and co-workers also reported the filling of graphitized carbon fibers (GNFs) and herringbone carbon nanofibers (CNFs) with pre-formed $\mathrm{Mn}_{3} \mathrm{O}_{4}$ MNPs. ${ }^{[72]}$ The different arrangement of the graphitic 


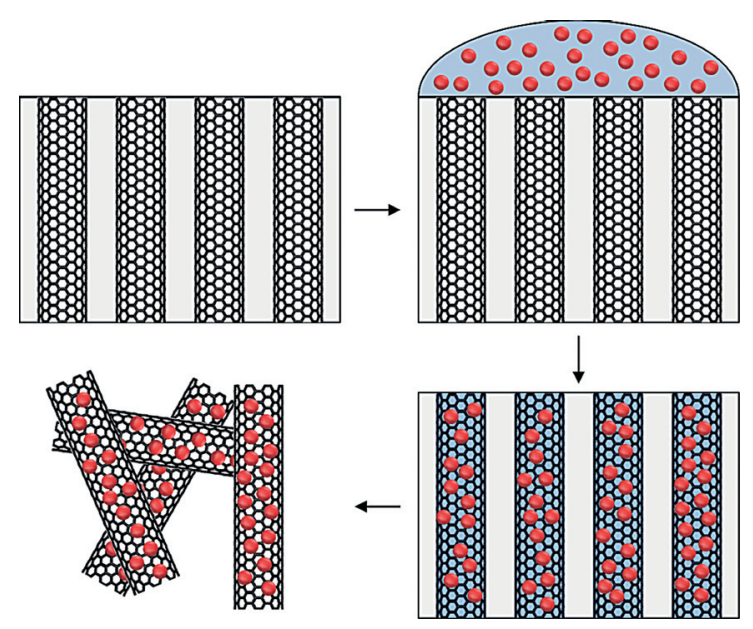

Figure 6. Schematic representation of the filling of CNTs with MNPs as performed by Korneva et al. ${ }^{[40]}$ CNTs are immobilized on a alumina support and a ferrofluid containing the MNPs is brought into contact and interfaced to a permanent magnetic field gradient. This allows an invasion of the pores, ultimately giving rise to the MNPs@CNTs after removal of the solvent and of the matrix. Adapted with permission from reference [40].

layers in the GNFs and CNFs dictated the arrangement of the inner $\mathrm{Mn}_{3} \mathrm{O}_{4}$ MNPs, thus giving hybrid materials (MNPs@GNF and MNPs@CNF) featuring different magnetic responses. For instance, the alignment of $\mathrm{Mn}_{3} \mathrm{O}_{4}$ MNPs governed by the stepedges of a GNF framework decreases the interparticle interactions, favoring saturation magnetization at lower fields.

\subsubsection{Exohedral functionalization}

Similarly to the ex-situ endohedral functionalization methods, coating of external surface of CNTs with MNPs (i.e., "exohedral" functionalization) can be achieved either by grafting preformed MNPs or by preparing MNPs on the nanotube surface. In both cases it is necessary to decorate the exo-surface of CNTs by different anchoring groups apt for immobilizing either MNPs or their precursors. This pre-functionalization can be either covalent or noncovalent, and the interested reader is addressed to the dedicated reviews in the field. ${ }^{[12 a, 73]}$ In this respect, Correa-Duarte et al. synthesized $\left(\mathrm{Fe}_{2} \mathrm{O}_{3}-\mathrm{Fe}_{3} \mathrm{O}_{4}\right) / C N T$ hybrids by the use of a layer-by-layer approach. ${ }^{[41]}$ Firstly, MWCNTs were coated with an anionic poly(sodium 4-styrene sulfonate) acting as a primer coat for the homogeneous adsorption of cationic poly(dimethyldiallylammonium) chloride, the latter acting as an anchoring surface for the negatively charged $\mathrm{Fe}_{2} \mathrm{O}_{3}-\mathrm{Fe}_{3} \mathrm{O}_{4}$ MNPs. Similarly, Stoffelbach et al. ${ }^{[74]}$ have decorated carboxylated-functionalized CNTs with positively charged $\mathrm{Fe}_{3} \mathrm{O}_{4}$ MNPs through electrostatic interactions. In an elegant approach, Georgakilas et al. ${ }^{[75]}$ prepared pyrene-based carboxylic acid as a supramolecular anchor that, through hydrophobic interactions, on one side adheres on the graphitic surface and on the other electrostatically binds a positively charged MNP, like $\mathrm{Fe}_{3} \mathrm{O}_{4}$, Co, or CoPt MNP. Using oleic acid, an improved solubility in organic solvents was achieved, allowing for a better processability of the MNP/CNT supramolecular hybrid.
On another hand, it is possible to grow MNPs on the surface of CNTs. For example, in 2007 Jia et al. ${ }^{[76]}$ adsorbed $\mathrm{Fe}^{3+}$ cations onto negatively-charged oxidized MWCNTs. Reducing the chemisorbed $\mathrm{Fe}^{3+}$ atoms by addition of polyethyleneglycol under heating conditions at $200^{\circ} \mathrm{C}$ for $8-12 \mathrm{~h}$, yielded $5 \mathrm{~nm}$ $\mathrm{Fe}_{3} \mathrm{O}_{4}$ MNPs that, in turn, acted as a potential secondary seed for growing other $\mathrm{Fe}_{3} \mathrm{O}_{4}$ MNPs. Recent use of the hydrothermal process was reported by $\mathrm{Yu}$ et al. ${ }^{[77]}$ Their procedure consists in the addition of $\mathrm{H}_{2} \mathrm{O}_{2}$ to an aqueous mixture of CNTs and $\mathrm{FeSO}_{4}$. The mixture of $\mathrm{H}_{2} \mathrm{O}_{2}$ and $\mathrm{Fe}^{2+}$ (Fenton's reagent) forms $\mathrm{Fe}^{3+}$ and hydroxyl radicals able to oxidize CNTs that can interact with $\mathrm{Fe}^{3+}$ cations. After precipitation and subsequent reduction with $\mathrm{H}_{2}$ at $450^{\circ} \mathrm{C}, \mathrm{Fe}_{2} \mathrm{O}_{3}$ MNPs were uniformly formed on the CNT surface.

\section{3. "Magnetic" Synergies between CNTs and MNPs}

Merging MNPs and CNTs gives birth to hybrids with physicochemical and structural properties that greatly differ from the mere addition of the characteristics proper to the single components (Figure 7). In particular, the cavity-confinement of MNPs holds great potentials for all the magnetic applications listed above, especially those for biotechnology, as the magnetic material is shielded from the external environment. Thanks to the work of Kappen et al. ${ }^{[78]}$ it was shown that encapsulated $\alpha$-Fe remains unaltered for more than 2.5 years, while unprotected Fe MNPs are oxidized in a matter of seconds to a few minutes. ${ }^{[79]}$ This further preserves the metallic $\alpha$ Fe that, in comparison to the oxidized forms, possesses higher magnetic saturation values (171 $\mathrm{emu}^{-1}$ compared to 76 and 92 emu g $^{-1}$ for $\gamma-\mathrm{Fe}_{2} \mathrm{O}_{3}$ and $\mathrm{Fe}_{3} \mathrm{O}_{4}$, respectively), ${ }^{\left[{ }^{[0]}\right.}$ which are desirable for magnetic applications like hyperthermia and sorting. It is worth noting that MNP@CNT hybrids, prepared by exsitu methods, also contain important amounts of non-oxidized particles thus suggesting that even open-tips CNTs confer a protective action on the encapsulated particles. In this respect, Tessonier et al. ${ }^{[68]}$ demonstrated that confined CoFe particles can be protected from oxidation reactions, whereas unencapsulated particles are oxidized in air at room temperature in a few minutes. In their experiment, CoFe@CNT hybrids (synthesized by reduction of $\mathrm{CoFe}_{2} \mathrm{O}_{4} @ C N T$ precursors, cf. Section 2.2.1) were heated stepwise in air under variable temperature (from room temperature to $500^{\circ} \mathrm{C}$ ), while monitoring with powder X-ray diffraction. No oxidation of CoFe MNPs occurred before $200^{\circ} \mathrm{C}$, while complete conversion in $\mathrm{CoFe}_{2} \mathrm{O}_{4}$ was only obtained at $350^{\circ} \mathrm{C}$. It was postulated that the high aspect ratio of CNTs somehow prevents $\mathrm{O}_{2}$ diffusion inside the tubes, hampering extensive oxidation at room temperature.

Beside the structural protective action, the hollow graphitic structure can also be used as a non-inert cavity allowing for the transformation of reactive chemical species. For instance, it was demonstrated that oxidized MNPs like $\mathrm{Fe}_{2} \mathrm{O}_{3}$ can be thermally reduced (from $585^{\circ} \mathrm{C}$ ) inside CNTs, yielding metallic Fe phases through a reductive process involving the graphitic layers tangentially contacting the oxide structures, ${ }^{[81]}$ while exohedrally coated $\mathrm{Fe}_{2} \mathrm{O}_{3}$ MNPs require higher temperatures 


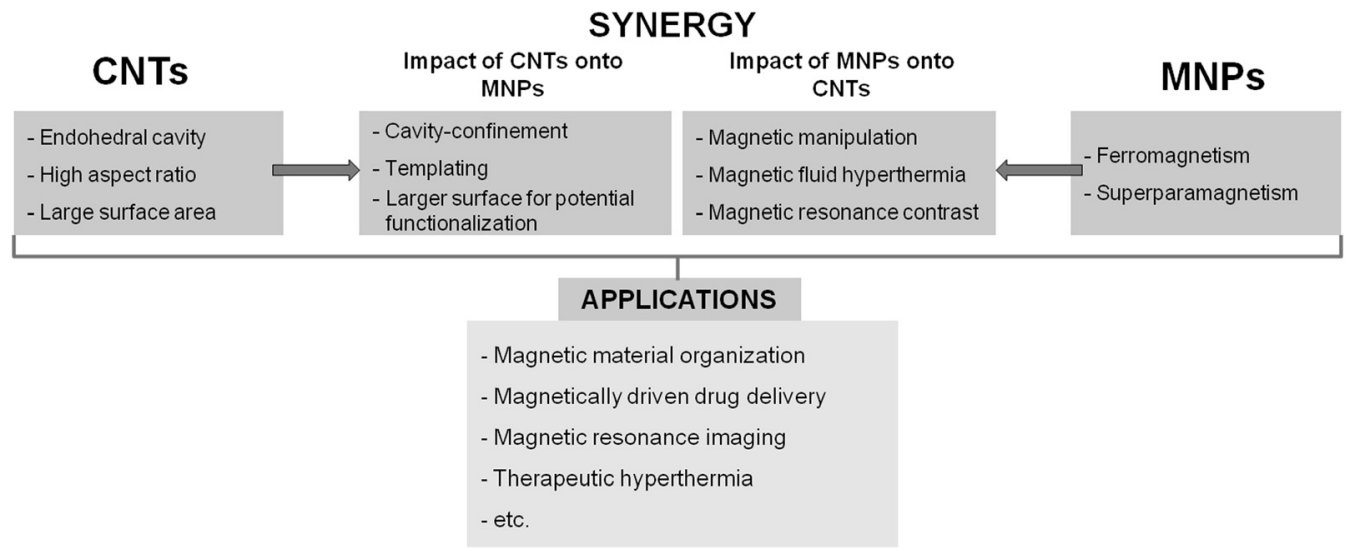

Figure 7. Chemical and physical synergies between CNTs and MNPs.

$\left(800^{\circ} \mathrm{C}\right)$. Small-diameter CNTs $(2-4 \mathrm{~nm})$ favored the reductive transformation. In addition, by applying the in-situ methodology, the nanotube framework was exploited as a template to mold MNPs into rods. This morphology is more appealing than that of spherical particles of the same diameter, since rods display higher coercivity ${ }^{[29]}$ due to the increase of the total magnetic anisotropy energy, which contributes to keep the magnetic moments aligned. ${ }^{[38]}$ In this regard, very high values (430 to $1070 \mathrm{Oe}$ ) for encapsulated Fe nanorods $(5-40 \mathrm{~nm}$ outer diameter and $10 \mu \mathrm{m}$ in length) were measured by Grobert et al., ${ }^{[82]}$ which is one to two order of magnitude higher than bulk Fe. The shape effect was further demonstrated by Shi et al., ${ }^{[83]}$ who succeeded to tune the coercivity of Fe@MWCNTs by controlling the aspect ratio of the encapsulated Fe nanowires. Changing the aspect ratio of the Fe rods from 1.6 to 6.0 caused a coercivity enhancement, from 300 to 800 Oe.

Other magnetic parameters can be influenced by the cavityconfinement. In this regard, Zhao and co-workers reported an increase of the magnetic moment of confined Ni MNPs by a factor of $3.4( \pm 1.0) .{ }^{[84]}$ Few years later, the same group reported an enhancement of the magnetic moment of encapsulated $\mathrm{Fe}$ and $\mathrm{Fe}_{3} \mathrm{O}_{4}$ in MWCNTs also by a factor of three. ${ }^{[85]}$ Although some hypotheses have been proposed to explain these observations, investigations are still needed to fully rationalize this phenomenon.

The synergy between MNPs and CNTs can also take place during the synthesis. As discussed above, the inner cavity of CNTs can act as a "nano-reactor" and template the synthesis of monodisperse Co NPs. ${ }^{[66]}$ On the other hand, MNPs can also be used to govern the growth of different types of CNTs. An original example of this concept was reported by Wei et al. ${ }^{[86]}$ By introducing a magnet above or beside the substrate during the CVD process, one can tune the coalescence or the division of the Fe catalyst particles during the CVD process, giving rise to either branched or linear CNTs (Figure 8). This protocol is called "magnetism-assisted CVD".

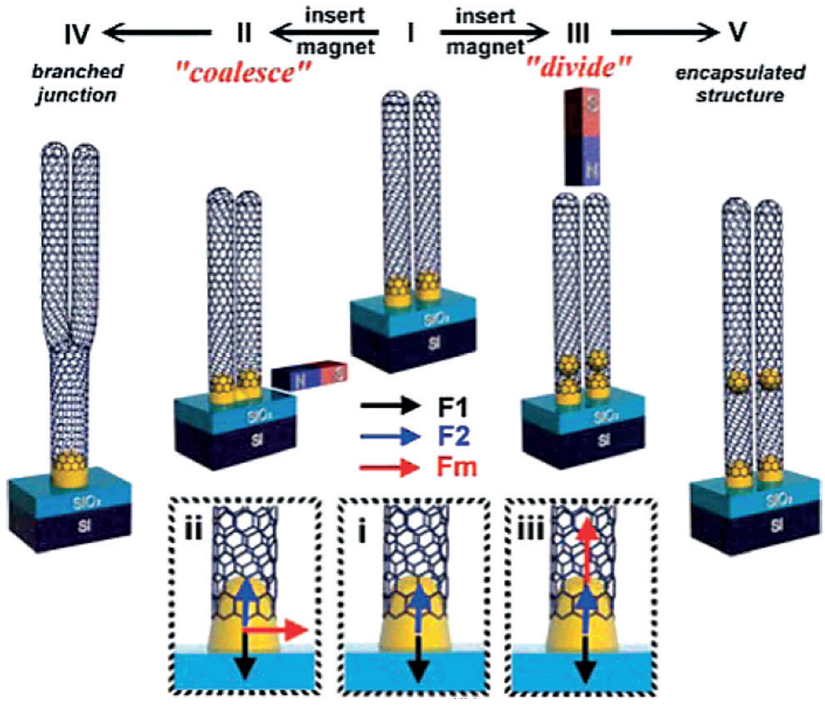

Figure 8. Schematic representation of the "magnetism-assisted CVD" protocol. $^{[86]}$ The coalescence or the separation of the magnetic Fe particles can be achieved by placing the magnet beside or above the substrate, leading to branched or Fe-filled CNTs. Reproduced with permission from reference [86].

\section{Applications of Magnetically Active Carbon Nanotubes}

In this section, we describe the main applications of the magnetically active CNTs. The chapter is structured in such a way that the examples are grouped following the action exerted by the presence of an external magnetic field, namely: 1) movement (rotation or translation), 2) heat production and 3) contrast generation.

\subsection{Magnetically guided motions}

Magnetic fields can determine two different responses of MNPs-CNTs in terms of motion: rotations and translations. These two different motions have been exploited for both material- and biomedical-oriented applications. 


\subsubsection{Rotational movements}

Translating the unique nanoscale properties of CNTs to the bulk as in a macroscopic device remains a difficult challeng $\mathrm{e}^{[87]}$ and a solution to this problem consists in controlling the assembly/spatial organization of CNTs, and in particular their alignment. ${ }^{[88]}$ This allows for the convergence of individual CNT features, inducing a sort of synergistic effect on materials properties. $^{[89]}$ Specifically, aligned CNTs form a better conductive corridor for electrons and phonons, dramatically enhancing the thermal and electrical conductivities. ${ }^{[90]}$ Therefore, a common approach to enhance the properties of materials would be to dope organic matrixes (like films, gels and polymers) with aligned CNTs. ${ }^{[23]}$ For instance, a polymer matrix containing aligned CNTs was revealed to possess enhanced mechanical strength in that direction, due to the fact that the stress is distributed along the CNT's main axis. ${ }^{[90 a, 91]}$ The intrinsic magnetic susceptibility anisotropy of CNTs was exploited to induce, through the application of an external magnetic field, the alignment of CNTs blended inside polymeric matrixes. ${ }^{[22]}$ However, the need of high magnetic field strengths (around 10 T) makes this approach quite inconvenient, as it requires very expensive set-ups and thus is limited to low-scale productions. However, if one decorates CNTs with MNPs, alignment of CNTs can be achieved at room temperature with an external magnetic field as low as $0.2 \mathrm{~T}$ (Figure 9). ${ }^{[41]}$ Following this approach, Kim et al. ${ }^{[93]}$ succeeded in aligning CNTs in polymer matrixes. In
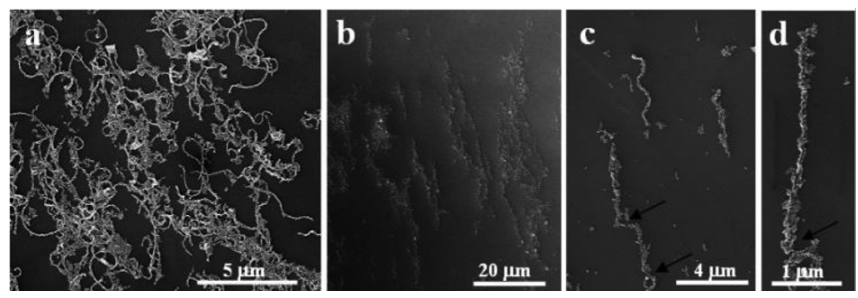

Figure 9. SEM images of MNPs/CNTs in a) the absence and b-d) the presence of an external magnetic field. The arrows indicate the presence of head-to-tail CNTs connection to form chain-like structures. Reproduced with permission from reference [41].

detail, they synthesized $\gamma-\mathrm{Fe}_{2} \mathrm{O}_{3} / \mathrm{CNT}$ hybrids by a sol-gel method and embedded them inside an epoxy polymer. $\mathrm{A}$ weak $0.3 \mathrm{~T}$ magnetic field revealed to be sufficient for aligning the $\mathrm{Fe}_{2} \mathrm{O}_{3} / C N T$ hybrids during the formation of the material as proven by TEM analyses. The anisotropic distribution of CNTs efficiently improved the electrical conductivity of the polymer, with an anisotropic enhancement of about 4 times along the direction of the magnetic field than perpendicularly to it.

Rotational motion of MNPs-CNTs can also be used for biological perspectives. As a proof of concept, Gao et al. ${ }^{\left[{ }^{[4]}\right.}$ used $\mathrm{Fe}_{3} \mathrm{O}_{4} / \mathrm{CNT}$ hybrids as nano-tools for manipulation of sheep blood cells. In particular, they showed that their positively charged hybrids were able to bind to the cells and that, upon application of a rotating magnetic field $\left(12.7 \mathrm{KAm}^{-1}\right.$ at a frequency of $0.5 \mathrm{~Hz}$ ), a rotational motion of single cells or pairs of cells bridged together could be achieved as displayed in Figure 10.

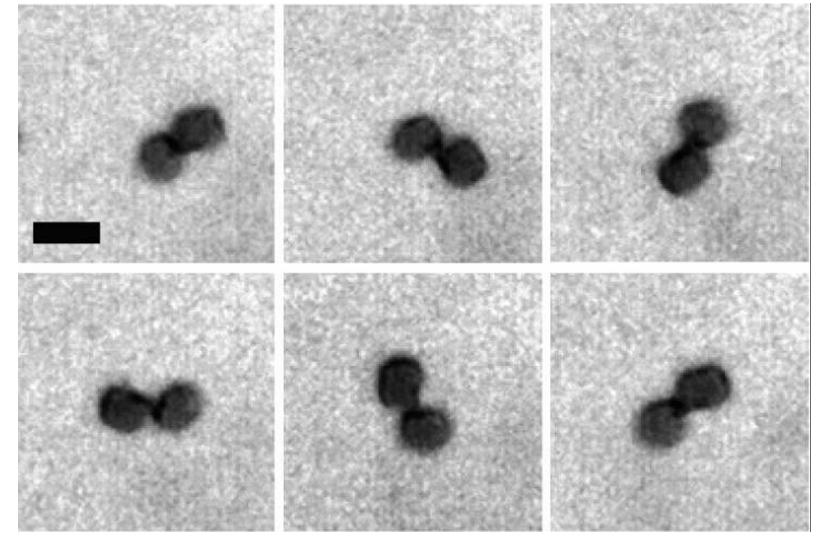

Figure 10. Sequence of the rotational motion of sheep blood cells bridged with MNPs/CNTs in a magnetic field of $12.7 \mathrm{KA} \mathrm{m}^{-1}$ and at a frequency of $0.5 \mathrm{~Hz}$. The snapshots correspond to $0,0.2,0.4,0.6,0.8$, and 1 st cycle of the rotational magnetic field. Reproduced with permission from reference [94].

\subsubsection{Translation}

Beside the alignment of MNPs-CNTs in homogeneous directional magnetic fields due to a torque, a magnetic field gradient can drive the translation of MNPs-CNTs toward a desired position and along a given path. This property can be used to recover MNPs-CNTs or to specifically guide the hybrids toward a particular target (as for example in in-vivo drug-delivery). As an example, our group reported the possibility to magnetically recycle a polymer-coated Fe@CNT hybrid used to purify wastewater. ${ }^{[95]}$ Raw Fe@CNT hybrids were produced by Fc-catalyzed CVD and then coated with a poly(vinylpyridine) (PVP) polymer. In the presence of divalent metal ions (such as $\mathrm{Zn}^{2+}, \mathrm{Cu}^{2+}$, and $\mathrm{Pb}^{2+}$ ) contained in wastewater, the hybrid formed insoluble bundles in which the $\mathrm{M}^{2+}$ cations remained trapped into pyrid$\mathrm{yl}-\mathrm{M}^{2+}$-pyridyl interactions. In the presence of a permanent magnet, these bundles could be magnetically recovered, along with the polluting $\mathrm{M}^{2+}$ cations (Figure 11). By this method, up
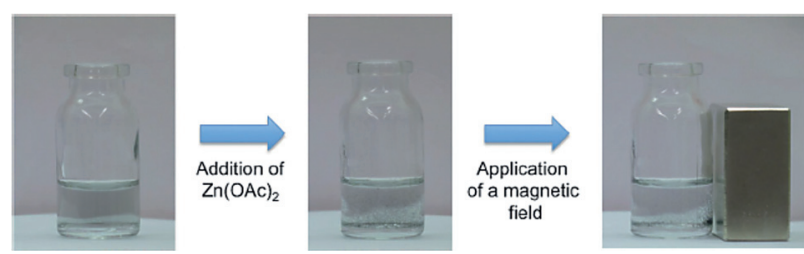

Figure 11. Purification of water using MNPs@CNTs. A stable suspension of m-PVPy-MNPs@CNTs (left) precipitates upon addition of $\mathrm{Zn}(\mathrm{OAc})_{2}$ (middle) and can be separated from the solution with a permanent magnet (right) thus purifying the solution. Reproduced with permission from reference [95].

to $99 \%$ of the initial amount of contaminants were removed. Moreover, a subsequent acidic treatment released these cations to recycle the depolluting agent. Other examples of magnetic recovery using MNP-CNT hybrids include the $\mathrm{pH}$-dependent removal of $\mathrm{Co}^{2+}$ cations performed by Wang et al. ${ }^{[96]}$ and the sequestration of cationic dyes by Gong et al. ${ }^{[97]}$ These two studies used oxidized CNTs coated with iron oxide NPs. 
Translation of MNPs-CNTs upon magnetic fields gradients holds also a great importance for biomedical applications. In that respect, Cai et al. have demonstrated that CNTs (previously studied $^{[98]}$ for intracellular delivery of drugs, ${ }^{[18,99]}$ proteins, ${ }^{[19]}$ or nucleic acids ${ }^{[98 e, 100]}$ ) uptake by cells can be dramatically enhanced by magnetic spearing process. ${ }^{[101]}$ In their study, they showed that weakly magnetic MNPs@CNTs (encapsulating residual amounts of $\mathrm{Ni}$ ) can spear the cell membrane with the aid of a rotating magnetic field. In a second step, the speared cells were deposited on a substrate and a static magnetic field was applied beneath, pulling the MNPs@CNTs through the membrane inside the cytoplasm (Figure 12). By this protocol,

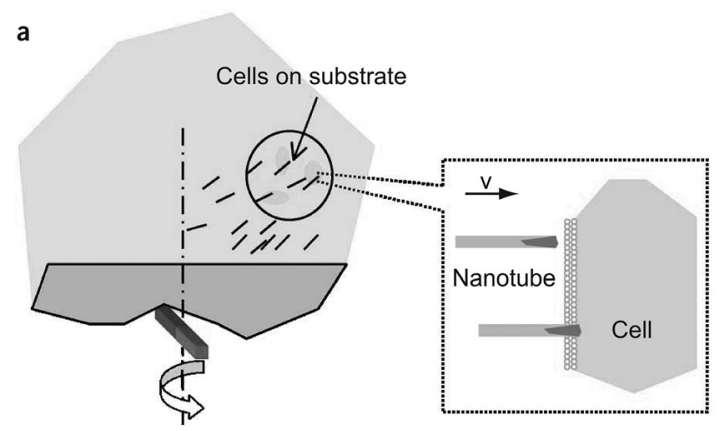

b

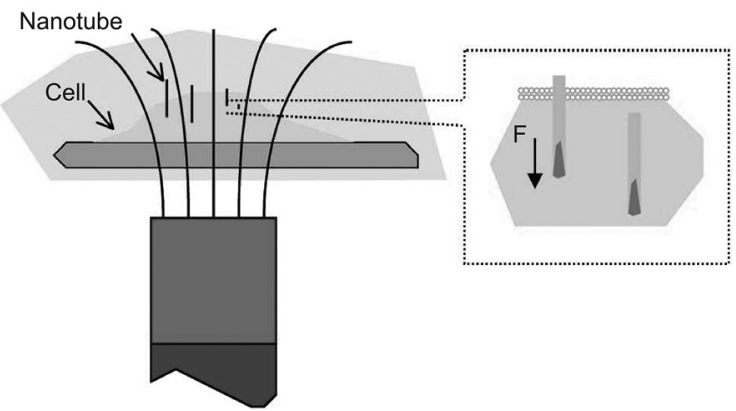

Figure 12. The two-steps procedure ${ }^{[101]}$ for spearing cells membranes with MNPs@CNTs. a) In the first step, a rotating magnetic field drives CNTs (black lines) to spear the cells by penetrating the membrane; b) in a second stage, a static field is used to pull CNTs inside the cells. Reproduced with permission from reference [101].

the authors succeeded to efficiently transduce DNA plasmids, coding for the fluorescent green protein, inside B-lymphoma and neuronal cells. It is worth noting that only very small (femtomolar) concentrations of MNPs@CNTs are needed for an efficient transduction. In comparison, non-magnetic transduction of non-magnetic CNTs requires micromolar ${ }^{[19]}$ to nanomolar ${ }^{[100]}$ concentrations. In the same vein, Liu et al. very recently demonstrated the possibility to use amino-functionalized Co and Fe oxides MNPs@CNTs, produced from the stearate precursors method developed earlier by the same team ${ }^{[66]}$ (Figure 5), for magnetic manipulation of cells in-vitro. ${ }^{[102]}$ They were able to show that, depending on the direction and the frequency of the field applied, their hybrids can be either taken up by cells, drive cell motion, or act as a remote "nano-stirring bar" in the biological media.
Functionalization with targeting agents such as antibodies bestows specific cell-recognition properties on CNTs. ${ }^{[103]}$ By associating this approach with the concept of magnetic filtration, our group demonstrated the possibility of magnetic separation of targeted cancer cells over other cell lines by Fe@CNTs conjugated to the monoclonal antibody Cetuximab (Figure 13). ${ }^{[104]}$
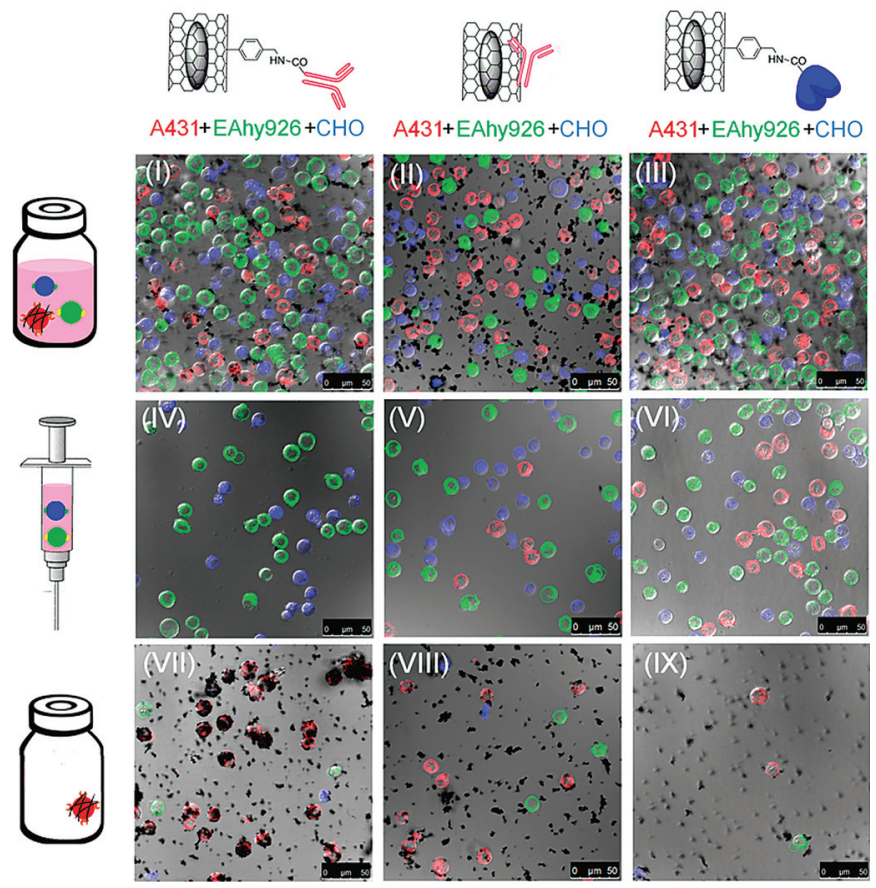

Figure 13. Confocal microscopy images of cell suspensions interfaced with Fe@CNTs bearing covalently linked Cetuximab (I, IV and VII), Fe@CNTs/Cetuximab physical mixture (II, V, VIII) or Fe@CNTs covalently functionalized with the reference protein BSA, which lacks of targeting properties. EGFR + cells (A431 cells) were stained with red-fluorescent dye, while EGFR - cells were stained with green (Eahy926 cells) or far-red ( $\mathrm{CHO}$ cells, blue coloration) dyes, respectively. Magnetic sorting steps: mixing of three differently stained cell-lines, addition of functionalized MNPs@CNTs and slight agitation to allow the binding (I-III). Magnetic filtration and subsequent separation of the supernatant, containing the EGFR- cells (IV-VI) from the magneticallycoagulated EGFR + cells (VII-IX). Scale bars $50 \mu \mathrm{m}$. Reproduced with permission from reference [104].

We proved that the conjugation with Cetuximab is responsible for the ability of our graphitic material to discriminate between EGFR + cancer cells (overexpressing epidermal growth factor receptors) from EGFR - model lines. It is worth noting that the magnetic separation occurred in a very short timescale (in the order of few minutes) opening future perspectives for this hybrid as an efficient circulating cancer cells removal agent.

Additionally, magnetically induced translation of MNPs-CNTs can be used for in-vivo magnetic delivery in murine models. In this respect, Fu and co-workers reported in a pilot study the possibility to target lymph nodes by MNPs/CNTs. ${ }^{[105]}$ Importantly, neither toxicity nor CNT aggregation in other organs were observed. Subsequently, the same group developed this technology, using the static magnetic field of a permanent magnet positioned in direct contact with the targeted organs, to provide the magnetically driven in-vivo delivery of a drug (gemcitabine) loaded on MNPs/MWCNTs. ${ }^{[106]}$ 


\subsection{Heat production through magnetic fluid hyperthermia}

In another avenue, CNTs have recently attracted lot of attention due to their high absorbance in the IR spectral region, which makes them excellent agents for IR-based hyperthermia anti-cancer treatments. ${ }^{[103 a, 107]}$ Magnetic fluid hyperthermia ${ }^{[37]}$ (MFH) is another complementary approach in which MNPs are heated by application of an alternating magnetic field. A major advantage of MFH over other hyperthermic methods relies on the use of relatively low frequencies (in the $\mathrm{kHz}$ range, compared to the $\mathrm{THz}$ range of infrared waves), which generally pass harmlessly through the whole body and heat only tissues containing MNPs. ${ }^{[37]}$ The feasibility of MFH with MNPs@CNTs was first reported by Krupskaya et al. who studied low-loaded (4 wt\% in Fe) Fe@CNTs produced by CVD. ${ }^{[108]}$ Employing a magnetic field of $80 \mathrm{kAm}^{-1}$ and a frequency of $139 \mathrm{kHz}$, they were able to show a temperature increase of more than $10^{\circ} \mathrm{C}$ after 6 min for Fe@CNTs dispersed in water at a concentration of $5 \mathrm{mg} \mathrm{mL}^{-1}$. No temperature increase was observed for the purified MWCNTs under the same conditions.

Employing bioconjugated Fe@CNTs bearing Cetuximab mentioned above ${ }^{[104]}$ (cf. Section 4.1.2.), our group also demonstrated that MNPs@CNTs could have a moderate, but selective, magnetic fluid hyperthermia activity. Upon application of an alternating magnetic field of $83 \mathrm{kAm}^{-1}$ and $220 \mathrm{kHz}$ for $15 \mathrm{~min}$ onto a mixture of EGFR + and EGFR - cells, it was possible to observe a moderate yet significant increased cytotoxicity for the EGFR + cellular population (20-25\% cell death) in comparison with that of the EGFR- cells (7-8\% cell death). The selective MFH effect is presumably due to an agglomeration of Fe@CNT-Cetuximab hybrids around the EGFR + cells, maximizing the local Fe concentration responsible for the enhanced MFH effect. Recent results also showed that depending on the Fe phases (e.g., $\alpha$-Fe or Fe carbide) and loading, different MFH and translational responses were observed. ${ }^{[109]}$

\subsection{Contrast agents in magnetic resonance imaging}

The use of CNTs for in-vivo applications as drug carrier ${ }^{[106]}$ or agent for hyperthermia therapy ${ }^{[110]}$ requires a careful monitoring of their biodistribution. Thanks to their physical properties, the fate of in-vivo injected CNTs can be followed using Raman spectroscopy, photo-acoustic imaging or near-infrared fluorescence microscopy. ${ }^{[111]}$ One can also functionalize CNTs with radioisotopes allowing single-photon-emission computed tomography (SPECT) or positron emission tomography (PET). ${ }^{[12]}$ Each of these techniques has advantages and limitations. For instance, the spectroscopic techniques using low-wavelength electromagnetic waves possess a good resolution, but have limitations in tissue-depth penetration, whereas the detection of radioisotopes has the advantage of being very sensitive, but involves ionizing radiation.

On the other hand, magnetic resonance imaging (MRI) is a very versatile and non-invasive technique based on the nuclear magnetic resonance of protons contained in constituents of the body, such as water and lipids. ${ }^{[113]}$ Magnetic spins of protons are oriented in a magnetic field (1 to $10 \mathrm{~T}$ ) and excited with an appropriate resonant radio-frequency. The MRI signal originates from the emission of another radio-frequency during protons de-excitation. In particular, the rates of de-excitation depend on the environment of the proton and are characteristic of a particular chemical structure and, thus, of a tissue. This is the origin of the contrast observed in a classical MRI image. De-excitation is measured by relaxation times, either longitudinal $\left(T_{1}\right)$ or transverse $\left(T_{2}\right)$. These values are the decay constants for the longitudinal and transversal components (with respect to the magnetic field) of the protons nuclear spin magnetization. The versatility of MRI relies on the fact that its signal depends on multiple physicochemical parameters such as $\mathrm{pH}$, density of protons, oxygenation level, flow rates, and so forth. However, MRI is intrinsically a low-sensitivity method due to the small energy difference between the two spin states of protons $\left(4.26 \times 10^{-26} \mathrm{~J}\right.$ in a magnetic field of 1.5 T) and often requires the use of contrast agents (CAs). In this methodology, the contrast enhancement ${ }^{[114]}$ relies on the decrease of the $T_{1}$ and $T_{2}$ values of protons in close proximity, leading to hyper-intensity ("white") zones in $\mathrm{T}_{1}$-weighted images and hypo-intensity ("black") zones in $\mathrm{T}_{2}$-weighted images. ${ }^{[115]}$ Among the existing $\mathrm{CAs}, \mathrm{Gd}^{3+}$ holds a prominent place due to its very large magnetic moment $\left(\mu^{2}=63 \mu_{\mathrm{B}}^{2}\right) \cdot{ }^{[16]}$ However, the significant toxicity limits its use and chelated $\mathrm{Gd}^{3+}$ ions are typically used. ${ }^{[116]}$ Wilson and co-workers encapsulated for the first time $\mathrm{Gd}^{3+}$ ions inside SWCNTs (called "gadonanotubes"). ${ }^{[17]}$ Remarkably, the cavity confinement not only decreased the toxicity of the $\mathrm{Gd}^{3+}$ ions, but also greatly enhanced the $T_{1}$ relaxivity (by a factor of $40: 170 \mathrm{vs} .4 \mathrm{~mm}^{-1} \mathrm{~s}^{-1}$ at $1.5 \mathrm{~T}$, with the latter being the common value for classical $T_{1}$-CAs). ${ }^{[118]}$

MNPs represent another class of MRI CAs, reducing principally $T_{2}$ relaxation times by induction of proton spin dephasing by energy exchange between the atomic nuclei. ${ }^{[35,120]}$ As a consequence, MRI appears as a "natural" solution to track MNPsCNTs in-vivo allowing the combination of therapeutic applications with imaging techniques. In 2007, Choi et al. reported one of the first seminal studies on the potentials of MNPsCNTs for MRI applications. ${ }^{[120 b]}$ They used magnetically enriched SWCNTs possessing $3 \mathrm{~nm}$ iron oxide NPs at one of their extremities (iron loading of $35 \mathrm{wt} \%$ and a magnetic saturation of $56 \mathrm{emu} \mathrm{g}^{-1}$ ). A supramolecular coating with a 30-mer DNA sequence imparted individualization and water solubilization to their $\mathrm{Fe}_{2} \mathrm{O}_{3} / \mathrm{CNT}$ hybrids. Measurements of the $T_{1}$ and $T_{2}$ gave a magnetic relaxation timescale ratio $\left(T_{1} / T_{2}\right)$ of approximately 12 , which is in the range of the other reported MNP CAs (215). This was further exploited in dual imaging mode analysis, in which near-IR fluorescence spectroscopy was used to complement MRI. In another report, Yang and co-workers developed a new approach to evaluate the uptake ratio of MNPsCNTs incubated with cells. ${ }^{[119]}$ For this purpose, they synthesized $\mathrm{CoFe}_{2} \mathrm{O}_{4} / \mathrm{CNTS}$ displaying a $T_{2}$ relaxivity $\left(R_{2}\right)$ of $152.8 \mathrm{~mm}(\mathrm{Fe})^{-1} \mathrm{~s}^{-1}$ in the usual range of other MNPs used as CAs (i.e., $100-400 \mathrm{~mm}^{-1} \mathrm{~s}^{-1}$ ). ${ }^{[121]}$ The contrast effect of this hybrid allowed them to correlate the decrease of $T_{2}$ observed in incubated cancer HeLa cells with the amount of the internalized $\mathrm{CoFe}_{2} \mathrm{O}_{4} @$ CNTs (Figure 14). More recently, Jia and co-work- 
(a) $\mathrm{HeLa}$
MWCNT/CoFe
$\mathrm{O}_{4}-180$ $\mu \mathrm{gL}^{-1}$

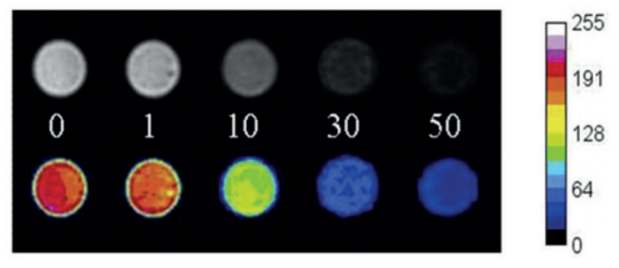

(c)

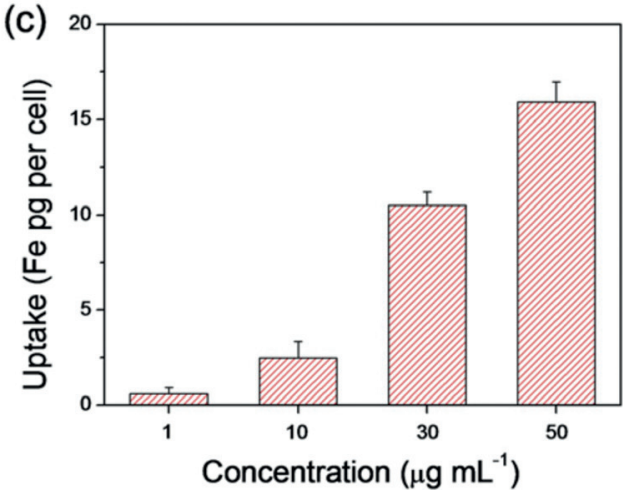

(b)

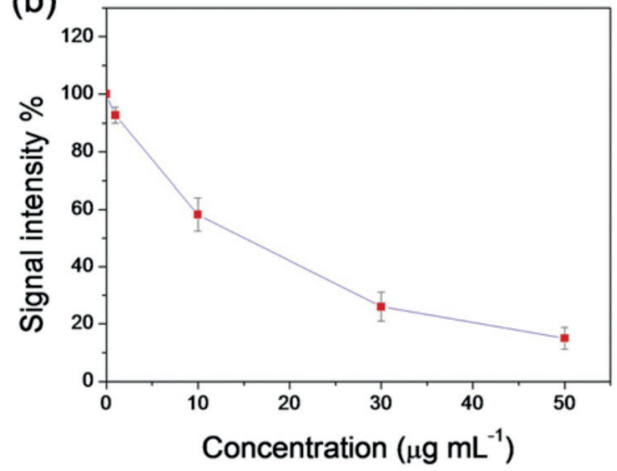

Figure 14. a) In-vitro $T_{2}$-weighted MR images of HeLa cells treated with different concentrations of $\mathrm{CoFe}_{2} \mathrm{O}_{4} /$ MWCNTs (in $\mu \mathrm{g} \mathrm{mL}^{-1}$ ). b) Signal intensity percentage of the labeled cells compared to untreated cells as a function of the concentration of $\mathrm{CoFe}_{2} \mathrm{O}_{4} /$ MWCNTs. c) Iron uptake in the cells as a function of the concentration of $\mathrm{CoFe}_{2} \mathrm{O}_{4} /$ MWCNTs. Reproduced with permission from reference [119].

ers also reported the synthesis of water-dispersible $\mathrm{Fe}_{2} \mathrm{O}_{3} / \mathrm{CNTS}$ functionalized with folic acid. ${ }^{[122]}$ By using HeLa cells overexpressing folate receptors, the authors also demonstrated a different MRI response upon targeting.

MNPs-CNTs have also been exploited for in-vivo applications. In this respect, Al-Faraj et al. assessed the pulmonary toxicity of inhaled non-purified and purified HiPco SWCNTs (produced by in-situ decomposition at high pressure of $\mathrm{CO}$ and $\mathrm{Fe}(\mathrm{CO})_{5}$, containing residual amounts of Fe MNPs) in rat models, ${ }^{[123]}$ by combining hyperpolarized ${ }^{3} \mathrm{He}$ and ${ }^{1} \mathrm{H}$ MRI. The susceptibility effect induced by the non-purified Fe-SWCNTs was sufficient to provoke a significant drop in the magnetic field homogeneity as detected by ${ }^{3} \mathrm{He}$ MRI that, combined with ${ }^{1} \mathrm{H}$ MRI and histological analyses, permitted the determination of the dose-dependent granulomatous and inflammatory reactions in the lungs caused by Fe-SWCNTs. ${ }^{[123]}$ The same group also combined ${ }^{1} \mathrm{H}$ MRI analysis with Raman spectroscopy ${ }^{[124]}$ to assess biodistribution of the intravenously injected Fe-SWCNTs, showing a preferential accumulation in liver and spleen. Along the same lines, Al-Jamal and co-workers reported the ex-situ synthesis of radioisotope-labeled $\mathrm{Fe}_{2} \mathrm{O}_{3}$ MWCNTs for dual SPECT and MR imaging. ${ }^{[125]} \mathrm{Fe}_{2} \mathrm{O}_{3}$ MNPs, which were used to exohedrally coat MWCNTs, were functionalized with the radioisotope ${ }^{99 \mathrm{~m}} \mathrm{Tc}$ through a bisphosphonate linker. In particular, SPECT allowed a whole-body imaging and quantitative biodistribution analysis, which is difficult to attain solely with MRI. On the other hand, MRI allowed a high-resolution mapping of the distribution in organs such as liver (Figure 15).

\section{Conclusion and Perspectives}

In this article, we describe the concept of merging CNTs and MNPs to give birth to magnetically active MNPs-CNTs hybrids, which, depending on the spatial association, can be grouped in two families: exohedral or endohedral derivatives. These materials feature magnetic properties that derive from a synergistic association of the individual constituents. For instance, marrying $\mathrm{Fe}_{2} \mathrm{O}_{3}$ NPs to CNTs gives magnetically active hybrids that, under weak external fields (ca. 0.3 T), can be aligned to engineer anisotropically organized materials. Similarly, the possibility to remotely pilot these graphitic scaffolds by an external magnetic field gradient, such as that originating from a commercially available permanent magnet, opens the way to several applications in which these hybrids are used as catchers to clear solutions from inorganic pollutants or physiological medium from biological bodies like cancer cells. Magnetically driven translational movements can also be exploited to locally guide and deliver drugs both in-vitro and in-vivo. In addition, MNPs confer to the CNTs hybrids the possibility to locally generate heat under the stimulus of a remote alternating magnetic field or to act as a $T_{2}$-contrast agent in MRI. Among the different hybrids, cavity-confined MNPs are particularly interesting as the magnetic material is shielded from oxidation reactions, thus

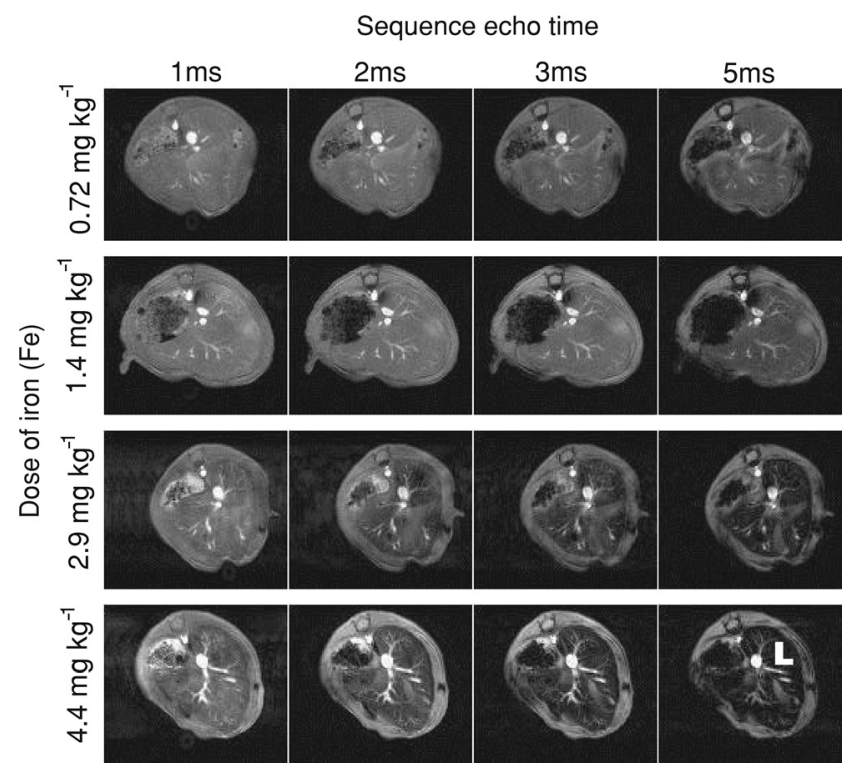

Figure 15. In-vivo $T_{2}^{*}$ weighted MR images of mouse liver $(\mathrm{L}) 48 \mathrm{~h}$ after intravenous injections of different doses of ${ }^{99 \mathrm{~m}} \mathrm{Tc}$-radiolabeled $\mathrm{Fe}_{2} \mathrm{O}_{3}$ /MWCNTs. A darkening of the liver is observable upon increase of the injected dose, due to higher liver concentration of $\mathrm{Fe}_{2} \mathrm{O}_{3}$ /MWCNTs acting as $T_{2}{ }^{*}$ contrast agents. Reproduced with permission from reference [125]. 
featuring higher magnetic saturations overtime. However, the full exploitation of the synergistic properties of these hybrid materials is still far from being fully exhausted. For instance, suitable insulating surfaces coated with carpets of highly-coercive MNPs@CNTs could be exploited for the design of new magnetic storage devices featuring a high density of information per surface area. Additionally, it has been shown that the organization of MNPs plays a great role in the expression of macroscopic properties through the modification of their magnetic interactions. This could inspire the engineering of highlyorganized MNP/CNT hybrids featuring tunable magnetic properties, the characteristics of which would result from their peculiar arrangement.

\section{Acknowledgements}

This work was supported by the Belgian National Research Foundation (FRFC contract $n^{\circ}$ 2.4.550.09), the "TINTIN" ARC project from the Belgian French community (Contract No. 09/ 14-023), the Wallonia Region (THERAPLUS project), the Science Policy Office of the Belgian Federal Government (BELSPO-IAP 7/05 project), the EU through the ERC Starting Grant "COLORLANDS" and the FP7 NMP small collaborative project "SACS", and the MIUR (FIRB Futuro in Ricerca SUPRACARBON, contract no. RBFR10DAK6). R.M. and F.P. thank the Fund for Scientific Research (FNRS) for his postdoctoral and FRIA doctoral fellowships, respectively.

Keywords: carbon nanotubes - magnetic nanoparticles magnetic properties $\cdot$ nano-hybrids

[1] S. lijima, Nature 1991, 354, 56-58.

[2] X. Wang, Q. Li, J. Xie, Z. Jin, J. Wang, Y. Li, K. Jiang, S. Fan, Nano Lett. 2009, 9, 3137-3141.

[3] M. M. J. Treacy, T. W. Ebbesen, J. M. Gibson, Nature 1996, 381, 678 680.

[4] M.-F. Yu, O. Lourie, M. J. Dyer, K. Moloni, T. F. Kelly, R. S. Ruoff, Science 2000, 287, 637-640.

[5] T. W. Ebbesen, H. J. Lezec, H. Hiura, J. W. Bennett, H. F. Ghaemi, T. Thio, Nature 1996, 382, 54-56.

[6] H. Kataura, Y. Kumazawa, Y. Maniwa, I. Umezu, S. Suzuki, Y. Ohtsuka, Y. Achiba, Synth. Met. 1999, 103, 2555-2558.

[7] E. Pop, D. Mann, Q. Wang, K. Goodson, H. Dai, Nano Lett. 2006, 6, $96-$ 100.

[8] S. Sinha, S. Barjami, G. Iannacchione, A. Schwab, G. Muench, J. Nanopart. Res. 2005, 7, 651-657.

[9] Z. Spitalsky, D. Tasis, K. Papagelis, C. Galiotis, Prog. Polym. Sci. 2010, 35, $357-401$.

[10] X. Lu, Z. Chen, Chem. Rev. 2005, 105, 3643-3696.

[11] a) "Functionalization and Applications of [60]Fullerene": A. MateoAlonso, D. Bonifazi, M. Prato, in Carbon Nanotechnology (Ed.: L. Dai), Elsevier, Amsterdam, 2006, Chapter 7, pp. 155-189; b) J. L. Delgado, N. Martín, P. De La Cruz, F. Langa, Chem. Soc. Rev. 2011, 40, 5232-5241; c) F. Diederich, R. Kessinger, Acc. Chem. Res. 1999, 32, 537-545; d) M. Prato, M. Maggini, Acc. Chem. Res. 1998, 31, 519-526.

[12] a) D. Tasis, N. Tagmatarchis, A. Bianco, M. Prato, Chem. Rev. 2006, 106, $1105-1136$; b) N. Karousis, N. Tagmatarchis, D. Tasis, Chem. Rev. 2010, 110, 5366-5397.

[13] a) A. Krueger, in Carbon Materials and Nanotechnology, Wiley-VCH, Weinheim, 2010, Chapter 3; b) Carbon Nanotechnology (Ed.: L. Dai), Elsevier, Amsterdam, 2006; c) M. Meyyappan, in Carbon Nanotubes: Science and Applications, CRC, Boca Raton, 2004.
[14] R. Klingeler, R. B. Sim, in Carbon Nanotubes for Biomedical Applications, Springer, Berlin, 2011.

[15] S. J. Tans, A. R. M. Verschueren, C. Dekker, Nature 1998, 393, 49-52.

[16] P. Avouris, M. Freitag, V. Perebeinos, Nat. Photonics 2008, 2, 341-350.

[17] a) D. R. Kauffman, A. Star, Angew. Chem. Int. Ed. 2008, 47, 6550-6570; Angew. Chem. 2008, 120, 6652-6673; b) J. Wang, Electroanalysis 2005, $17,7-14$.

[18] A. Bianco, K. Kostarelos, M. Prato, Curr. Opin. Chem. Biol. 2005, 9, 674679.

[19] N. W. S. Kam, H. Dai, J. Am. Chem. Soc. 2005, 127, 6021-6026.

[20] N. W. S. Kam, Z. Liu, H. Dai, J. Am. Chem. Soc. 2005, 127, 12492-12493.

[21] a) H. Ajiki, T. Ando, J. Phys. Soc. Jpn. 1993, 62, 2470-2480; b) H. Ajiki, T. Ando, Physica B 1994, 201, 349-352; c) H. Ajiki, T. Ando, J. Phys. Soc. Jpn. $1995,64,4382-4391$.

[22] a) A. S. Kotosonov, J. Exp. Theor. Phys. 1999, 70, 476-480; b) M. Fujiwara, E. Oki, M. Hamada, Y. Tanimoto, I. Mukouda, Y. Shimomura, J. Phys. Chem. A 2001, 105, 4383-4386; c) J. Shaver, A. N. G. Parra-Vasquez, S. Hansel, O. Portugall, C. H. Mielke, M. Von Ortenberg, R. H. Hauge, M. Pasquali, J. Kono, ACS Nano 2009, 3, 131-138; d) S. Zaric, G. N. Ostojic, J. Kono, J. Shaver, V. C. Moore, R. H. Hauge, R. E. Smalley, X. Wei, Nano Lett. 2004, 4, 2219-2221; e) T. Searles, Y. Imanaka, T. Takamasu, H. Ajiki, J. Fagan, E. Hobbie, J. Kono, Phys. Rev. Lett. 2010, 105, 017403; f) J. P. Lu, Phys. Rev. Lett. 1995, 74, 1123-1126; g) M. A. Marques, M. D'avezac, F. Mauri, Phys. Rev. B 2006, 73, 125433; h) S. Zaric, G. N. Ostojic, J. Kono, J. Shaver, V. C. Moore, M. S. Strano, R. H. Hauge, R. E. Smalley, X. Wei, Science 2004, 304, 1129-1131.

[23] a) J. E. Fischer, W. Zhou, J. Vavro, M. C. Llaguno, C. Guthy, R. Haggenmueller, M. Casavant, D. Walters, R. E. Smalley, J. Appl. Phys. 2003, 93, 2157-2163; b) J. Hone, M. C. Llaguno, N. M. Nemes, A. T. Johnson, J. E. Fischer, D. A. Walters, M. J. Casavant, J. Schmidt, R. E. Smalley, Appl. Phys. Lett. 2000, 77, 666-668; c) B. Smith, Z. Benes, D. Luzzi, J. Fischer, D. Walters, M. Casavant, J. Schmidt, R. Smalley, Appl. Phys. Lett. 2000 $77,663-665$.

[24] J. M. Coey, in Magnetism and Magnetic Materials, Cambridge University Press, Cambridge, 2010.

[25] K. J. Buschow, F. R. De Boer, in Physics of Magnetism and Magnetic Materials, Springer, New York, 2003.

[26] P. Atkins, in Physical Chemistry, 8th ed., Oxford University Press, Oxford, 2006.

[27] Where, $M$ and $H$ are expressed as $A m^{-1}$. However the CGS (Centimeter-gram-second) units system is still the most employed nowadays. In this system, $M$ and $H$ have units of respectively emu cm ${ }^{-3}$ $\left(1 \mathrm{emu} \mathrm{cm}^{-3}=4 \pi / 1000 \mathrm{~A} \mathrm{~m}^{-1}\right)$ and Oe $\left(1 \mathrm{Oe}=1 / 1000 \mathrm{Am}^{-1}\right) \cdot \chi$ is either dimensionless (in SI) or expressed in emu cm ${ }^{-3}$ or emu cm ${ }^{-3} \mathrm{Oe}^{-1}$.

[28] Classical absolute values of molar $\chi$ for diamagnetic materials are around $10^{-6}$ to $10^{-5} \mathrm{emumol}^{-1}$ for $\mathrm{H}_{2} \mathrm{O}$ and those of paramagnetic material such as aluminum, or oxygen are around $10^{-5}$ to $10^{-3} \mathrm{emumol}^{-1}$. Some lanthanide salts such as those of gadolinium can however possess $\chi$ values up to $10^{-1}$ emu mol $^{-1}$.

[29] C. M. Sorensen, Nanoscale Materials in Chemistry (Ed.: K. J. Klabunde), Wiley-Interscience, 2001, Chapter 6, pp. 203.

[30] L. Maggini, M. Liu, Y. Ishida, D. Bonifazi, Adv. Mater. 2013, 25, $2462-$ 2467.

[31] A. Aharoni, in Introduction to the Theory of Ferromagnetism, Clarendon, Oxford, 1996.

[32] a) A.-H. Lu, E. L. Salabas, F. Schüth, Angew. Chem. Int. Ed. 2007, 46, 1222-1244; Angew. Chem. 2007, 119, 1242-1266; b) Q. A. Pankhurst, J. Connolly, S. Jones, J. Dobson, J. Phys. D 2003, 36, R167.

[33] B. D. Terris, T. Thomson, J. Phys. D 2005, 38, R199-R222.

[34] A. Schätz, O. Reiser, W. J. Stark, Chem. Eur. J. 2010, 16, 8950-8967.

[35] R. Qiao, C. Yang, M. Gao, J. Mater. Chem. 2009, 19, 6274-6293.

[36] S. Laurent, S. Dutz, U. O. Haefeli, M. Mahmoudi, Adv. Colloid Interface Sci. 2011, 166, 8-23.

[37] C. S. S. R. Kumar, F. Mohammad, Adv. Drug Delivery Rev. 2011, 63, 789808.

[38] R. Krahne, L. Manna, G. Morello, A. Figuerola, in Physical Properties of Nanorods, Springer, Berlin, 2013, Chapter 5, pp. 133-213.

[39] a) G. Modugno, C. Menard-Moyon, M. Prato, A. Bianco, Br. J. Pharmacol. 2015, 172, 975-991; b) A. Masotti, A. Caporali, Int. J. Mol. Sci. 2013, 14, $24619-24642$. 
[40] G. Korneva, H. Ye, Y. Gogotsi, D. Halverson, G. Friedman, J.-C. Bradley, K. G. Kornev, Nano Lett. 2005, 5, 879-884.

[41] M. A. Correa-Duarte, M. Grzelczak, V. Salgueirino-Maceira, M. Giersig, L. M. Liz-Marzan, M. Farle, K. Sierazdki, R. Diaz, J. Phys. Chem. B 2005 109, 19060-19063.

[42] D. Bethune, C. Klang, M. De Vries, G. Gorman, R. Savoy, J. Vazquez, R. Beyers, Nature 1993, 363, 605-607.

[43] C. Journet, W. Maser, P. Bernier, A. Loiseau, M. L. De La Chapelle, D. L. S Lefrant, P. Deniard, R. Lee, J. Fischer, Nature 1997, 388, 756-758.

[44] T. Guo, P. Nikolaev, A. Thess, D. Colbert, R. Smalley, Chem. Phys. Lett 1995, 243, 49-54.

[45] M. José-Yacamán, M. Miki-Yoshida, L. Rendón, J. G. Santiesteban, Appl. Phys. Lett. 1993, 62, 657-659.

[46] K. Hernadi, A. Fonseca, J. Nagy, D. Bernaerts, A. Lucas, Carbon 1996 34, $1249-1257$.

[47] S. Fan, M. G. Chapline, N. R. Franklin, T. W. Tombler, A. M. Cassell, H. Dai, Science 1999, 283, 512-514.

[48] R. Sen, A. Govindaraj, C. Rao, Chem. Phys. Lett. 1997, 267, 276-280.

[49] L. Huang, X. Cui, B. White, S. P. O'Brien, J. Phys. Chem. B 2004, 108 $16451-16456$.

[50] S. Sinnott, R. Andrews, D. Qian, A. Rao, Z. Mao, E. Dickey, F. Derbyshire, Chem. Phys. Lett. 1999, 315, 25-30.

[51] C. P. Deck, K. Vecchio, Carbon 2006, 44, 267-275.

[52] M. Kumar, Y. Ando, J. Nanosci. Nanotechnol. 2010, 10, 3739-3758.

[53] C. Kittel, P. Mceuen, in Introduction to Solid State Physics, Wiley, New York, 1976

[54] K. Kuwana, K. Saito, Carbon 2005, 43, 2088-2095.

[55] B. Satishkumar, A. Govindaraj, R. Sen, C. Rao, Chem. Phys. Lett. 1998 293, 47-52.

[56] U. Weissker, S. Hampel, A. Leonhardt, B. Büchner, Materials 2010, 3, $4387-4427$.

[57] It is referred to magnetic saturation $\left(M_{\mathrm{S}}\right)$ when the maximum magnetization is reached, for instance $M_{\mathrm{S}}$ of $\alpha-\mathrm{Fe}$ is around 12 emu mol ${ }^{-1}$.

[58] a) F. C. Dillon, A. Bajpai, A. Koós, S. Downes, Z. Aslam, N. Grobert, Carbon 2012, 50, 3674-3681; b) Q. Liu, Z.-G. Chen, B. Liu, W. Ren, F. Li, H. Cong, H.-M. Cheng, Carbon 2008, 46, 1892-1902.

[59] J. Jang, H. Yoon, Adv. Mater. 2003, 15, 2088-2091.

[60] B. W. Smith, M. Monthioux, D. E. Luzzi, Nature 1998, 396, 323-324.

[61] a) A. N. Khlobystov, D. A. Britz, G. a. D. Briggs, Acc. Chem. Res. 2005, 38 901 -909; b) R. Marega, D. Bonifazi, New J. Chem. 2014, 38, $22-27$.

[62] a) P. M. Ajayan, T. W. Ebbesen, T. Ichihashi, S. Iijima, K. Tanigaki, H. Hiura, Nature 1993, 362, 522-525; b) S. C. Tsang, Y. K. Chen, P. J. F. Harris, M. L. H. Green, Nature 1994, 372, 159- 162.

[63] D. Ugarte, T. Stöckli, J. Bonard, A. Châtelain, W. De Heer, Appl. Phys. A 1998, 67, $101-105$

[64] H. Geng, X. Zhang, S. Mao, A. Kleinhammes, H. Shimoda, Y. Wu, O Zhou, Chem. Phys. Lett. 2004, 399, 109-113.

[65] E. Dujardin, T. Ebbesen, H. Hiura, K. Tanigaki, Science 1994, 265, 1850 1852.

[66] W. Baaziz, S. Begin-Colin, B. P. Pichon, I. Florea, O. Ersen, S. Zafeiratos, R. Barbosa, D. Begin, C. Pham-Huu, Chem. Mater. 2012, 24, 1549-1551.

[67] C. Pham-Huu, N. Keller, C. Estournes, G. Ehret, M. Ledoux, Chem Commun. 2002, $1882-1883$.

[68] J.-P. Tessonnier, G. Winé, C. Estournès, C. Leuvrey, M.-J. Ledoux, C. Pham-Huu, Catal. Today 2005, 102, 29-33.

[69] N. R. Jana, Y. Chen, X. Peng, Chem. Mater. 2004, 16, 3931-3935.

[70] C. L. Bishop, M. Wilson, J. Mater. Chem. 2009, 19, 2929-2939.

[71] C.-M. Tîlmaciu, B. Soula, A.-M. Galibert, P. Lukanov, L. Datas, J. González, L. F. Barquín, J. R. Fernández, F. González-Jiménez, J. Jorge, Chem. Commun. 2009, 6664-6666.

[72] M. D. C. Gimenez-Lopez, A. La Torre, M. W. Fay, P. D. Brown, A. N. Khlobystov, Angew. Chem. Int. Ed. 2013, 52, 2051-2054; Angew. Chem. 2013, 125, 2105-2108.

[73] V. Georgakilas, D. Gournis, V. Tzitzios, L. Pasquato, D. M. Guldi, M. Prato, J. Mater. Chem. 2007, 17, 2679-2694.

[74] F. Stoffelbach, A. Aqil, C. Jerome, R. Jerome, C. Detrembleur, Chem. Commun. 2005, 4532-4533.

[75] V. Georgakilas, V. Tzitzios, D. Gournis, D. Petridis, Chem. Mater. 2005, 17, $1613-1617$.

[76] B. Jia, L. Gao, J. Sun, Carbon 2007, 45, 1476-1481.
[77] F. Yu, J. Chen, L. Chen, J. Huai, W. Gong, Z. Yuan, J. Wang, J. Ma, J. Colloid Interface Sci. 2012, 378, 175-183.

[78] P. Kappen, A. Rider, P. J. Pigram, N. Brack, J. Phys. Chem. C 2011, 115, $21083-21087$

[79] K. Haneda, A. H. Morrish, Nature 1979, 282, 186-188.

[80] H. M. Lu, W. T. Zheng, Q. Jiang, J. Phys. D 2007, 40, 320-325

[81] W. Chen, X. Pan, X. Bao, J. Am. Chem. Soc. 2007, 129, $7421-7426$.

[82] N. Grobert, W. K. Hsu, Y. Q. Zhu, J. P. Hare, H. W. Kroto, D. R. M. Walton, M. Terrones, H. Terrones, P. Redlich, M. Ruhle, R. Escudero, F. Morales, Appl. Phys. Lett. 1999, 75, 3363-3365.

[83] C. X. Shi, H. T. Cong, J. Appl. Phys. 2008, 104, 034307-034307.

[84] J. Wang, P. Beeli, Y. Ren, G.-M. Zhao, Phys. Rev. B 2010, 82, 193410

[85] G.-M. Zhao, J. Wang, Y. Ren, P. Beeli, Nano Lett. 2013, 13, 2993-2996.

[86] D. Wei, Y. Liu, L. Cao, L. Fu, X. Li, Y. Wang, G. Yu, J. Am. Chem. Soc 2007, 129, 7364-7368

[87] R. Van Noorden, Nature 2011, 469, 14-16.

[88] X. Sun, T. Chen, Z. Yang, H. Peng, Acc. Chem. Res. 2013, 46, 539-549.

[89] a) K. Koziol, J. Vilatela, A. Moisala, M. Motta, P. Cunniff, M. Sennett, A Windle, Science 2007, 318, 1892-1895; b) M. Grzelczak, M. A. CorreaDuarte, V. Salgueirino-Maceira, B. Rodriguez-Gonzalez, J. Rivas, L. M Liz-Marzan, Angew. Chem. Int. Ed. 2007, 46, 7026-7030; Angew. Chem. 2007, 119, $7156-7160$.

[90] a) P. Goh, A. Ismail, B. Ng, Composites Part A 2014, 56, 103-126; b) M. Abdalla, D. Dean, M. Theodore, J. Fielding, E. Nyairo, G. Price, Polymer 2010, 51, 1614-1620.

[91] H. G. Chae, S. Kumar, Science 2008, 319, 908-909.

[92] a) E. S. Choi, J. S. Brooks, D. L. Eaton, M. S. Al-Haik, M. Y. Hussaini, H. Garmestani, D. Li, K. Dahmen, J. Appl. Phys. 2003, 94, 6034-6039; b) T. Kimura, H. Ago, M. Tobita, S. Ohshima, M. Kyotani, M. Yumura, Adv. Mater. 2002, 14, 1380-1383.

[93] I. T. Kim, A. Tannenbaum, R. Tannenbaum, Carbon 2011, 49, 54-61.

[94] C. Gao, W. Li, H. Morimoto, Y. Nagaoka, T. Maekawa, J. Phys. Chem. B 2006, 110, 7213-7220.

[95] L. Maggini, J.-M. Raquez, R. Marega, J. Jensen Ahrens, F. Pineux, F. Meyer, P. Dubois, D. Bonifazi, ChemSusChem 2013, 6, 367-373.

[96] Q. Wang, J. Li, C. Chen, X. Ren, J. Hu, X. Wang, Chem. Eng. J. 2011, 174, $126-133$.

[97] J.-L. Gong, B. Wang, G.-M. Zeng, C.-P. Yang, C.-G. Niu, Q.-Y. Niu, W.-J. Zhou, Y. Liang, J. Hazard. Mater. 2009, 164, 1517-1522.

[98] a) K. T. Al-Jamal, H. Nerl, K. H. Müller, H. Ali-Boucetta, S. Li, P. D. Haynes, J. R. Jinschek, M. Prato, A. Bianco, K. Kostarelos, A. E. Porter, Nanoscale 2011, 3, 2627-2635; b) A. Antonelli, S. Serafini, M. Menotta, C. Sfara, F. Pierigé, L. Giorgi, G. Ambrosi, L. Rossi, M. Magnani, Nanotechnology 2010, 21, 425101-425101; c) A. M. A. Elhissi, W. Ahmed, I. U. Hassan, V. R. Dhanak, A. D'Emanuele, J. Drug Delivery 2012, 837327; d) H. Jin, D. A. Heller, R. Sharma, M. S. Strano, ACS Nano 2009, 3, 149-158; e) N. W. S. Kam, Z. Liu, H. Dai, Angew. Chem. Int. Ed. 2006, 45, 577-581; Angew. Chem. 2006, 118, 591-595; f) K. Kostarelos, L. Lacerda, G. Pas torin, W. Wu, S. Wieckowski, J. Luangsivilay, S. Godefroy, D. Pantarotto, J.-P. Briand, S. Muller, M. Prato, A. Bianco, Nat. Nanotechnol. 2007, 2 $108-113$.

[99] S. Y. Madani, N. Naderi, O. Dissanayake, A. Tan, A. M. Seifalian, Int. J. Nanomed. 2011, 6, 2963-2979.

[100] M. A. Herrero, F. M. Toma, K. T. Al-Jamal, K. Kostarelos, A. Bianco, T. Da Ros, F. Bano, L. Casalis, G. Scoles, M. Prato, J. Am. Chem. Soc. 2009, $131,9843-9848$

[101] D. Cai, J. M. Mataraza, Z.-H. Qin, Z. Huang, J. Huang, T. C. Chiles, D. Carnahan, K. Kempa, Z. Ren, Nat. Methods 2005, 2, 449-454.

[102] X. Liu, I. Marangon, G. Melinte, C. Wilhelm, C. Ménard-Moyon, B. P. Pichon, O. Ersen, K. Aubertin, W. Baaziz, C. Pham-Huu, S. Bégin-Colin A. Bianco, F. Gazeau, D. Bégin, ACS Nano 2014, 8, 11290-11304.

[103] a) P. Chakravarty, R. Marches, N. S. Zimmerman, A. D. E. Swafford, P. Bajaj, I. H. Musselman, P. Pantano, R. K. Draper, E. S. Vitetta, Proc. Natl. Acad. Sci. USA 2008, 105, 8697-8702; b) E. Venturelli, C. Fabbro, O. Chaloin, C. Ménard-Moyon, C. R. Smulski, T. D. Ros, K. Kostarelos, M. Prato, Small 2011, 7, 2179-2187; c) C.-H. Wang, Y.-J. Huang, C.-W. Chang, W.-M. Hsu, C.-A. Peng, Nanotechnology 2009, 20, $315101-$ 315101

[104] R. Marega, F. De Leo, F. Pineux, J. Sgrignani, A. Magistrato, A. D. Naik, Y. Garcia, L. Flamant, C. Michiels, D. Bonifazi, Adv. Funct. Mater. 2013, 23, 3173-3184. 
[105] F. Yang, J. Hu, D. Yang, J. Long, G. Luo, C. Jin, X. Yu, J. Xu, C. Wang, Q. Ni, D. Fu, Nanomedicine 2009, 4, 317-330.

[106] F. Yang, C. Jin, D. Yang, Y. Jiang, J. Li, Y. Di, J. Hu, C. Wang, Q. Ni, D. Fu, Eur. J. Cancer 2011, 47, 1873-1882.

[107] J. T. Robinson, K. Welsher, S. M. Tabakman, S. P. Sherlock, H. Wang, R. Luong, H. Dai, Nano Res. 2010, 3, 779-793.

[108] Y. Krupskaya, C. Mahn, A. Parameswaran, A. Taylor, K. Kraemer, S Hampel, A. Leonhardt, M. Ritschel, B. Büchner, R. Klingeler, J. Magn. Magn. Mater. 2009, 321, 4067-4071.

[109] F. Pineux, R. Marega, A. Stopin, A. La Torre, Y. Garcia, E. Devlin, C. Michiels, A. Khlobystov, D. Bonifazi, submitted.

[110] H. K. Moon, S. H. Lee, H. C. Choi, ACS Nano 2009, 3, 3707-3713.

[111] Z. Liu, S. Tabakman, K. Welsher, H. Dai, Nano Res. 2009, 2, 85-120.

[112] A. Ruggiero, C. H. Villa, J. P. Holland, S. R. Sprinkle, C. May, J. S. Lewis, D. A. Scheinberg, M. R. Mcdevitt, Int. J. Nanomed. 2010, 5, 783-802.

[113] R. H. Hashemi, W. G. Bradley, C. J. Lisanti, in MRI: The Basics, Lippincott, Williams \& Wilkins, Philadelphia, 2012

[114] The performance of a CA is usually expressed under the form of its relaxativities, $R_{1}$ and $R_{2}$. Relaxativities are defined as the change in the relaxation rate of water protons induced by a certain concentration of the CA expressed as: $R_{i}=T_{i}-1 / T_{i 0}$ (in $\mathrm{M}^{-1} \mathrm{~s}^{-1}$ ) in which $i=1$ or $2 ; T_{i}$ is the longitudinal or transverse relaxation time in presence of a certain concentration of CA and $T_{i 0}$ is the longitudinal or transverse relaxation time of the medium without CA.

[115] A. E. Merbach, É. Tóth, in The Chemistry of Contrast Agents in Medical Magnetic Resonance Imaging, Wiley, New York, 2001.

[116] P. Caravan, J. J. Ellison, T. J. Mcmurry, R. B. Lauffer, Chem. Rev. 1999, 99, $2293-2352$
[117] B. Sitharaman, K. R. Kissell, K. B. Hartman, L. A. Tran, A. Baikalov, I. Rusakova, Y. Sun, H. A. Khant, S. J. Ludtke, W. Chiu, S. Laus, E. Toth, L. Helm, A. E. Merbach, L. J. Wilson, Chem. Commun. 2005, 3915-3917.

[118] Q. Ma, M. Jebb, M. F. Tweedle, L. J. Wilson, J. Mater. Chem. B 2013, 1 , $5791-5797$.

[119] H. Wu, G. Liu, X. Wang, J. Zhang, Y. Chen, J. Shi, H. Yang, H. Hu, S. Yang, Acta Biomater. 2011, 7, 3496-3504.

[120] a) C. Sun, J. S. H. Lee, M. Zhang, Adv. Drug Delivery Rev. 2008, 60, $1252-1265$; b) J. H. Choi, F. T. Nguyen, P. W. Barone, D. A. Heller, A. E. Moll, D. Patel, S. A. Boppart, M. S. Strano, Nano Lett. 2007, 7, 861-867.

[121] a) L. Babes, J. Jacques, L. Jeune, P. Jallet, J. Colloid Interface Sci. 1999 212, 474-482; b) H. B. Na, I. C. Song, T. Hyeon, Adv. Mater. 2009, 21 $2133-2148$; c) J. W. M. Bulte, D. L. Kraitchman, NMR Biomed. 2004, 17, 484-499.

[122] M. Yin, M. Wang, F. Miao, Y. Ji, Z. Tian, H. Shen, N. Jia, Carbon 2012, 50, 2162-2170.

[123] a) A. Al Faraj, K. Cieslar, G. Lacroix, S. Gaillard, E. Canet-Soulas, Y. Cremillieux, Nano Lett. 2009, 9, 1023-1027; b) A. Al Faraj, A. Bessaad, K. Cieslar, G. Lacroix, E. Canet-Soulas, Y. Crémillieux, Nanotechnology 2010, 21, 175103.

[124] A. Al Faraj, F. Fauvelle, N. Luciani, G. Lacroix, M. Levy, Y. Crémillieux, E. Canet-Soulas, Int. J. Nanomed. 2011, 6, 351.

[125] J. T.-W. Wang, L. Cabana, M. Bourgognon, H. Kafa, A. Protti, K. Venner A. M. Shah, J. K. Sosabowski, S. J. Mather, A. Roig, X. Ke, G. Van Tendeloo, R. T. M. De Rosales, G. Tobias, K. T. Al-Jamal, Adv. Funct. Mater. 2014, 24, 1880-1894.

Published online on May 28, 2015 